\title{
Direct transcriptional regulation of neuropilin-2 by COUP-TFII modulates multiple steps in murine lymphatic vessel development
}

\author{
Fu-Jung Lin, ${ }^{1}$ Xinpu Chen, ${ }^{1}$ Jun Qin, ${ }^{1}$ Young-Kwon Hong, ${ }^{2}$ Ming-Jer Tsai, ${ }^{1,3}$ and Sophia Y. Tsai1,3 \\ 1Department of Molecular and Cellular Biology, Baylor College of Medicine, Houston, Texas, USA. 2Department of Surgery and \\ Department of Biochemistry and Molecular Biology, Norris Comprehensive Cancer Center, Keck School of Medicine, University of Southern California, \\ Los Angeles, California, USA. ${ }^{3}$ Program in Developmental Biology, Baylor College of Medicine, Houston, Texas, USA.
}

\begin{abstract}
The lymphatic system plays a key role in tissue fluid homeostasis. Lymphatic dysfunction contributes to the pathogenesis of many human diseases, including lymphedema and tumor metastasis. However, the mechanisms regulating lymphangiogenesis remain largely unknown. Here, we show that COUP-TFII (also known as $\mathrm{Nr2f2}$ ), an orphan member of the nuclear receptor superfamily, mediates both developmental and pathological lymphangiogenesis in mice. Conditional ablation of COUP-TFII at an early embryonic stage resulted in failed formation of pre-lymphatic ECs (pre-LECs) and lymphatic vessels. COUP-TFII deficiency at a late developmental stage resulted in loss of LEC identity, gain of blood EC fate, and impaired lymphatic vessel sprouting. siRNA-mediated downregulation of COUP-TFII in cultured primary human LECs demonstrated that the maintenance of lymphatic identity and VEGF-C-induced lymphangiogenic activity, including cell proliferation and migration, are COUP-TFII-dependent and cell-autonomous processes. COUP-TFII enhanced the pro-lymphangiogenic actions of VEGF-C, at least in part by directly stimulating expression of neuropilin-2, a coreceptor for VEGF-C. In addition, COUP-TFII inactivation in a mammary gland mouse tumor model resulted in inhibition of tumor lymphangiogenesis, suggesting that COUP-TFII also regulates neo-lymphangiogenesis in the adult. Thus, COUP-TFII is a critical factor that controls lymphangiogenesis in embryonic development and tumorigenesis in adults.
\end{abstract}

\section{Introduction}

The lymphatic system is comprised of a network of vessels, mediating tissue fluid homeostasis, immune surveillance, and dietary fat absorption in the intestine. The lymphatic fluid flows unidirectionally and ultimately returns to venous circulation via the thoracic duct (1). Growing evidence reveals that malformation or dysfunction of this network contributes to the pathogenesis of many diseases, including lymphedema, lymphatic dysplasia, tumor metastasis, and inflammatory diseases (2).

Recent work using lineage tracing and gene targeting experiments in mice and live imaging of developing lymphatic vasculature in zebrafish have supported the model of the venous origin of the lymphatic system as first proposed by Florence Sabin in 1902 (3-6). During mammalian development, lymphatic ECs (LECs), derived from a specific location of the vein, bud off from the venous endothelium to form primary lymphatic sacs. These primitive sacs continue to sprout, proliferate, and migrate into the periphery and undergo maturation to generate the entire lymphatic vasculature network throughout the body. Transcription factor SRY box 18 (Sox18) appears to be one of the first molecular markers identified for the induction of LEC differentiation (7). At E9 in the mouse, the expression of Sox18 in the cardinal vein becomes polarized, which acts as a molecular switch to induce the expression of homeobox transcription factor Prox1, and initiates LEC specification. In mice deficient of Prox1, the ECs fail to commit to a LEC fate. Instead, these cells retain some features of blood vascular ECs (BECs) and fail to form the lymphatic vascular system. Conversely, ectopic

Conflict of interest: The authors have declared that no conflict of interest exists. Citation for this article: J Clin Invest. 2010;120(5):1694-1707. doi:10.1172/JCI40101. expression of Prox1 in cultured cells reprograms BECs into LECs, suggesting its critical role in LEC specification $(4,8,9)$.

Once a subpopulation of ECs on one side of the cardinal vein commits to the lymphatic lineage around E9.5-E10.5, lymphangiogenesis or the formation of nascent lymphatic vessels is directly or indirectly influenced by the growth factors present in the vicinity. Members of the VEGF family (VEGF-C and VEGF-D) secreted by adjacent tissues act through the lymphatic endothelial receptors, VEGF receptor and neuropilin $(\mathrm{Nrp})$ receptor, to promote LEC growth, migration, and survival (2). Binding of VEGF-C to VEGFR3 and/or VEGFR2 leads to receptor activation and autophosphorylation, which in turn promotes lymphangiogenesis (10). Complete ablation of Vegfc in mice results in the loss of lymphatic vasculature (11). Likewise, the mutation that inactivates VEGFR3 function leads to lymphatic hypoplasia and primary lymphedema in both the mouse and the human (12-14). In addition to VEGF receptors, the VEFG-C coreceptor Nrp2 has been shown to enhance VEGF-C binding to the VEGF receptor $(15,16)$. Nrp2-null mice fail to develop small lymphatic vessels and capillaries (17). Importantly, antagonizing Nrp2 function or blocking VEGFR3 signaling reduces tumor lymphangiogenesis and inhibits tumor metastasis (18-20). The genetic evidence suggests that the VEGF-C/VEGFR3/Nrp2 signaling axis is critical for embryonic and adult lymphangiogenesis.

Chicken ovalbumin upstream promoter-transcription factor II (COUP-TFII), a member of the nuclear receptor superfamily, has been shown to play a key role in organogenesis, cell fate determination, cell differentiation, angiogenesis, and metabolic homeostasis (21-23). Disruption of COUP-TFII in mice leads to early embryonic lethality, with defects in angiogenesis and cardiovascular development (23). In addition, using an EC-specific gene inactivation 
A
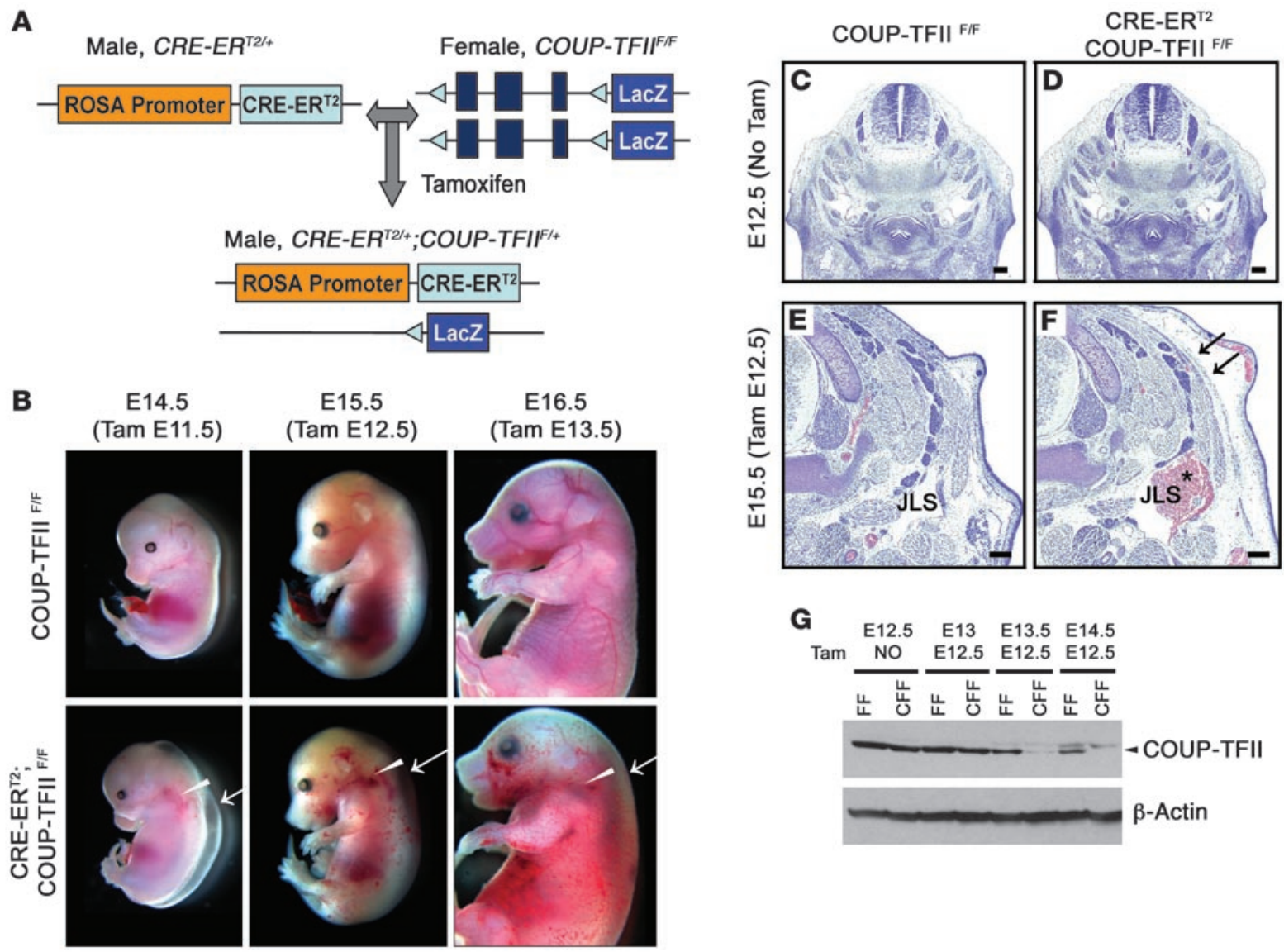

Figure 1

Conditional inactivation of COUP-TFII during embryogenesis leads to edema, hemorrhage, and blood-filled lymphatics. (A) Schematic representation of Cre-mediated COUP-TFII inactivation strategy. Embryos receive Tam via maternal intraperitoneal injection. (B) Whole-mount images of E14.5, E15.5, and E16.5 COUP-TFIF/F control and CRE-ER T2;COUP-TFIIF/F mutant embryos 3 days after Tam administration. Mutant embryos exhibit interstitial edema (arrows), hemorrhage (arrowheads), and some blood-filled dermal vessels. (C-F) H\&E-stained transverse sections of littermate controls and CRE-ER ${ }^{T 2}$;COUP-TFIF/F embryos at E12.5 without Tam induction (C and D) and at E15.5 (3 days after Tam treatment) (E and F). (F) Blood-filled lymphatics (asterisk) and interstitial edema (arrows) were observed in E15.5 CRE-ERT2; COUP-TFIIF/F embryos. JLS, jugular lymphatic sac. Scale bars: $200 \mu \mathrm{m}$. (G) Kinetics of CRE-ERT2-mediated COUP-TFII inactivation. Whole embryo lysates from embryos with $0,0.5$, 1 , and 2 days after Tam treatment were analyzed by Western blot analysis, using antibodies against COUP-TFIl and $\beta$-actin. The arrowhead indicates 47-kDa COUP-TFII protein. FF, COUP-TFIF/F control embryos; CFF, CRE-ER ${ }^{T 2}$; COUP-TFIIF/F mutant embryos. $n=3$ embryos per group.

system, we have demonstrated that COUP-TFII serves as a major regulator for the establishment of venous identity (22). Given that the mammalian lymphatic system has a venous origin (3), we asked whether the venous-derived lymphatic system requires COUP-TFII function during embryogenesis and adulthood.

Here, we demonstrate that the early development of lymphatic vasculature and LEC specification critically depends on COUPTFII. At a later developmental stage, conditional ablation of COUP-TFII in mice results in the malformation of primitive lymphatic sacs and lymphatic vessels. These LECs lose their identity and ectopically express BEC markers, even in the presence of Prox1 expression. The lymphatic vascular abnormalities are attributed to defective VEGFR3 signaling, resulting in reduced LEC growth, sprouting, and migration. This effect is mediated, at least in part, by COUP-TFII regulation of Nrp2 gene expression in a cell-autonomous function in lymphangiogenesis. While COUP-TFII does not seem to regulate the maintenance of adult lymphatics, inactivation of COUP-TFII in a mammary gland mouse tumor model results in an inhibition of tumor lymphangiogenesis, revealing an important role of COUP-TFII in neo-lymphangiogenesis in adults. These findings elucidate what we believe to be novel roles of COUP-TFII in modulating normal as well as pathological lymphangiogenesis.

\section{Results}

Conditional inactivation of COUP-TFII leads to edema, hemorrhage, and blood-filled vessels. Homozygous COUP-TFII mutants or ECspecific COUP-TFII knockout embryos die prior to E10.5 or E11.5, respectively, which precludes the assessment of the role of COUP-TFII in lymphatic physiology. To bypass early embryonic lethality and study the temporal functions of COUP-TFII during lymphatic vascular development, we conditionally inactivated COUP-TFII by crossing the COUP-TFII floxed mouse strain $\left(C O U P-T F I I^{F / F}\right)(24)$ with ROSA26CRE-ER ${ }^{T 2}$ (hereafter referred to as $C R E-E R^{T 2}$ ), encoding tamoxifen-inducible (Tam-inducible) Cre recombinase under the control of the ubiquitously active ROSA26 promoter (25). CRE-ER ${ }^{T 2}$;COUP-TFII ${ }^{F / F}$ males were inter- 

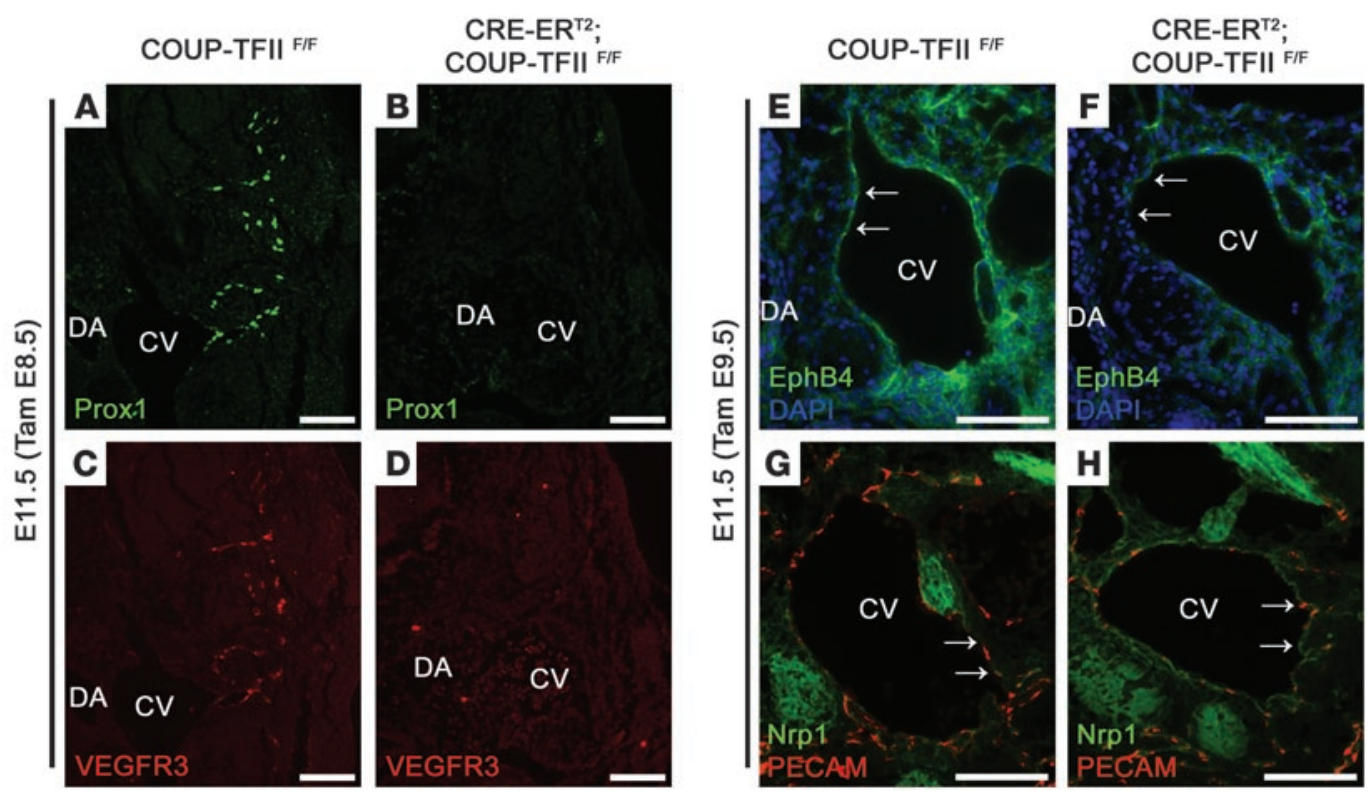

Figure 2

Failure of formation of pre-LECs in COUP-TFII mutants. (A-D) Transverse sections of control COUP-TFIIF/F and CRE-ER ${ }^{\text {T2 }}$;COUP-TFIF/F embryos at E11.5 (Tam administrated at E8.5) were analyzed using antibodies against Prox1 (A and B) and VEGFR3 (C and D). Prox1- and VEGFR3expressing LEC progenitors are visible in and around 1 side of the cardinal vein (CV) of E11.5 control COUP-TFIIF/F embryos (A and C) but are almost undetectable in CRE-ER $R^{T 2}$;COUP-TFIIF/F mutant embryos (B and D). DA, dorsal aorta. (E and F) Immunofluorescence shows that the expression of venous marker EphB4 is reduced in the cardinal vein of inducible COUP-TFII mutants at E11.5 (Tam administration at E9.5). ( $\mathbf{G}$ and $\mathbf{H})$ Nrp1 (green), an arterial marker, is ectopically expressed in the PECAM-positive (red) ECs of the cardinal vein of E11.5 COUP-TFII mutants (Tam administered at E9.5) but not controls. The arrows denote venous endothelium. Scale bar: $100 \mu \mathrm{m}$.

crossed with COUP-TFIIF/F females, and a single dosage of $3 \mathrm{mg}$ Tam was injected intraperitoneally into pregnant dams at various developmental stages to induce COUP-TFII deletion (Figure 1A). In the absence of Tam treatment, CRE-ER ${ }^{T 2}$; COUP-TFII ${ }^{F / F}$ embryos were indistinguishable from COUP-TFII ${ }^{F / F}$ control littermates (Figure 1, C and D). In contrast, injection of Tam into pregnant females, from E10.5 onward, activated the Cre recombinase, deleted the COUP-TFII gene, and caused the striking phenotypes of subcutaneous edema (Figure 1, B, E, and F), hemorrhages (Figure 1B), and blood-filled vessels (Figure 1F) in E13.5-E16.5 CRE-ER ${ }^{T 2}$;COUP-TFII ${ }^{F / F}$ embryos (Figure $1 \mathrm{~B}$ ).

To evaluate the kinetics of CRE-ER ${ }^{T 2}$-mediated COUP-TFII inactivation, a single dosage of $3 \mathrm{mg}$ Tam was injected intraperitoneally into pregnant dams at E12.5, and embryos were harvested at 0 , $0.5,1$, and 2 days after Tam treatment. Western blot analysis of whole embryo lysates and immunofluorescence analysis revealed that injection of a single dosage of $3 \mathrm{mg}$ Tam for 1 or 2 days was sufficient to ablate COUP-TFII, without affecting embryonic viability (Figure 1G and Supplemental Figure 1; supplemental material available online with this article; doi:10.1172/JCI40101DS1). In addition, allele excision analysis and COUP-TFII transcript analysis using DNA and mRNA, respectively, isolated from embryonic ECs sorted by FACS clearly showed that COUP-TFII is efficiently deleted in the ECs of the mutant embryos (Supplemental Figure 2).

A recent study showed that certain Tam-inducible CRE-ER ${ }^{T 2}$ mouse lines alone cause developmental defects indicating Cre toxicity (26). To exclude this possibility, we examined the formation of the cardiac lymphatic system in CRE-ER ${ }^{T 2}$ control embryos. We showed that CRE-ER ${ }^{T 2}$;COUP-TFII ${ }^{F /+}$ and CRE-ER ${ }^{T 2}$ controls are phenotypically indistinguishable from wild-type or COUP-TFII ${ }^{F /+}$ controls, in terms of gross appearance and cardiac lymphatic vascular patterning, as determined by whole-mount VEGFR3 immunostaining (Supplemental Figure 3), confirming that the developmental defects observed in inducible COUP-TFII knockout mutants are due to the ablation of COUP-TFII.

Failure offormation of pre-LECs in COUP-TFII mutants. We showed previously that COUP-TFII is specifically expressed in the venous but not in the arterial endothelium, and endothelial-specific deletion of COUP-TFII in mice resulted in the ectopic expression of arterial markers in the cardinal veins, indicating that COUP-TFII regulates venous identity (22). Subsequently, Srinivasan et al. showed a drastic reduction in the number of Prox1-expressing LEC precursors in and around the cardinal veins of endothelialspecific COUP-TFII knockout embryos at E11, suggesting that COUP-TFII may have a role in the formation of LECs (3). To determine whether COUP-TFII is required for lymphangiogenesis, we first asked whether conditional inactivation of the COUP-TFII gene subsequent to specification of veins alters venous identity. Tam was injected into pregnant females at E8.5, and embryos were harvested and analyzed at E11.5. The lymphatic and venous identities were examined at the molecular level, by evaluating the expression of lymphatic-specific genes and arterial-venous marker genes.

Around E10.5-E11, when the primary lymphatic plexus begins to form, Prox1-expressing LEC progenitors reside in and around one side of the cardinal vein (Figure $2 \mathrm{~A}$ ). These LEC progenitors also express other lymphatic markers, such as VEGF receptor 3 (VEGFR3) in the control (Figure 2C). However, no Prox1- or VEGFR3-positive cells were detected in the mutants lacking 

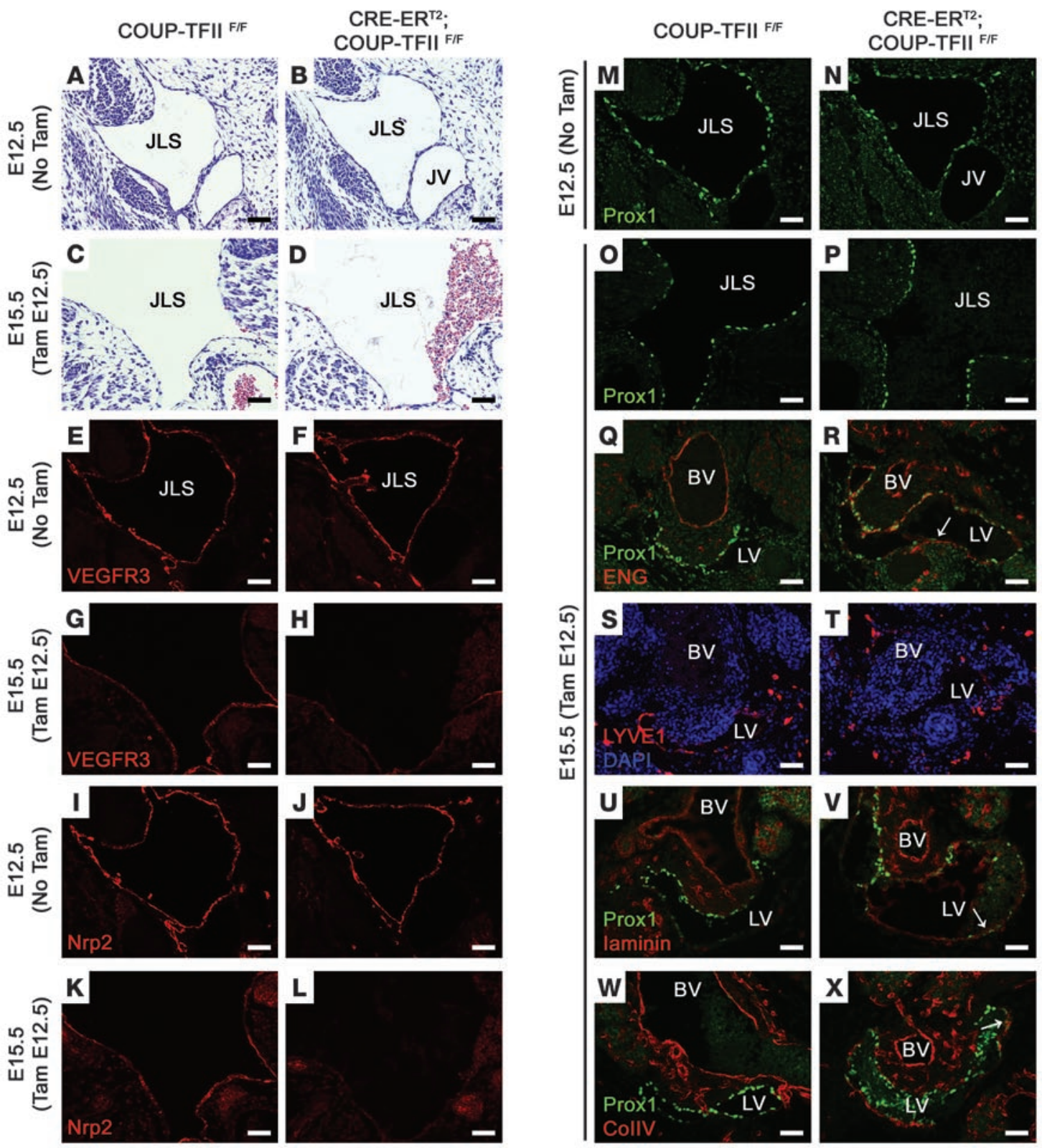

Figure 3

COUP-TFII regulates lymphatic vessel identity during embryogenesis. COUP-TFIl excision at a late developmental stage resulted in loss of LEC identity and gain of BEC fate. (A-P) H\&E staining and immunofluorescent images of transverse sections through the jugular region of control COUP-TFIIF/F and littermate CRE-ER ${ }^{T 2} ;$ COUP-TFIIF/F mutant embryos at E12.5 without Tam treatment $(\mathbf{A}, \mathbf{B}, \mathbf{E}, \mathbf{F}, \mathbf{I}, \mathbf{J}, \mathbf{M}$, and $\mathbf{N})$ and at E15.5 (with Tam administration at E12.5) (C, D, G, H, K, L, O, and P). VEGFR3 (E and F), Nrp2 (I and J), and Prox1 (M and N) are similarly expressed at E12.5 in controls and CRE-ER ${ }^{T 2}$;COUP-TFIFIF mutant embryos prior to Tam treatment. (G, $\mathbf{H}, \mathbf{K}$, and $\left.\mathbf{L}\right)$ Both VEGFR3 and Nrp2 are markedly reduced, however, at E15.5 in inducible COUP-TFII mutants compared with controls. (O and $\mathbf{P})$ Prox1 expression is unaffected in E15.5 inducible COUP-TFII mutants. (Q, R, and $\mathbf{U}-\mathbf{X})$ Blood vessel markers, endoglin (ENG), laminin, and collagen IV (CollV), are ectopically expressed in the Prox1-expressing lymphatic vessels (arrows) of E15.5 inducible COUP-TFIl mutants. ( $\mathbf{S}$ and $\mathbf{T}$ ) The expression of the lymphatic marker LYVE1 is significantly reduced in the lymphatic vessels of E15.5 inducible COUP-TFII mutants. BV, blood vessel; JV, jugular vein; LV, lymphatic vessel. Scale bars: $50 \mu \mathrm{m}$.

COUP-TFII (Figure 2, B and D). Similarly, Nrp2 and lymphatic vessel endothelial hyaluronan receptor 1 (LYVE1), 2 other lymphatic markers that were expressed in the sprouting lymphatic sacs in the controls, were also not detected in the COUP-TFII mutants (data not shown). When COUP-TFII deletion was induced at E9.5 and embryos were analyzed at E11.5, Prox1-, VEGFR3-, Nrp2-, and LYVE1-expressing LEC progenitors were detected only in the controls but not in the mutant embryos (Supplemental Figure 4). In contrast, the expression of Prox 1 in the non-EC type, such as sympathetic ganglia (Supplemental Figure 5, A and B), and neural tube (Supplemental Figure 5, C and D) was unaffected in the absence of COUP-TFII, suggesting that the lack of expression 


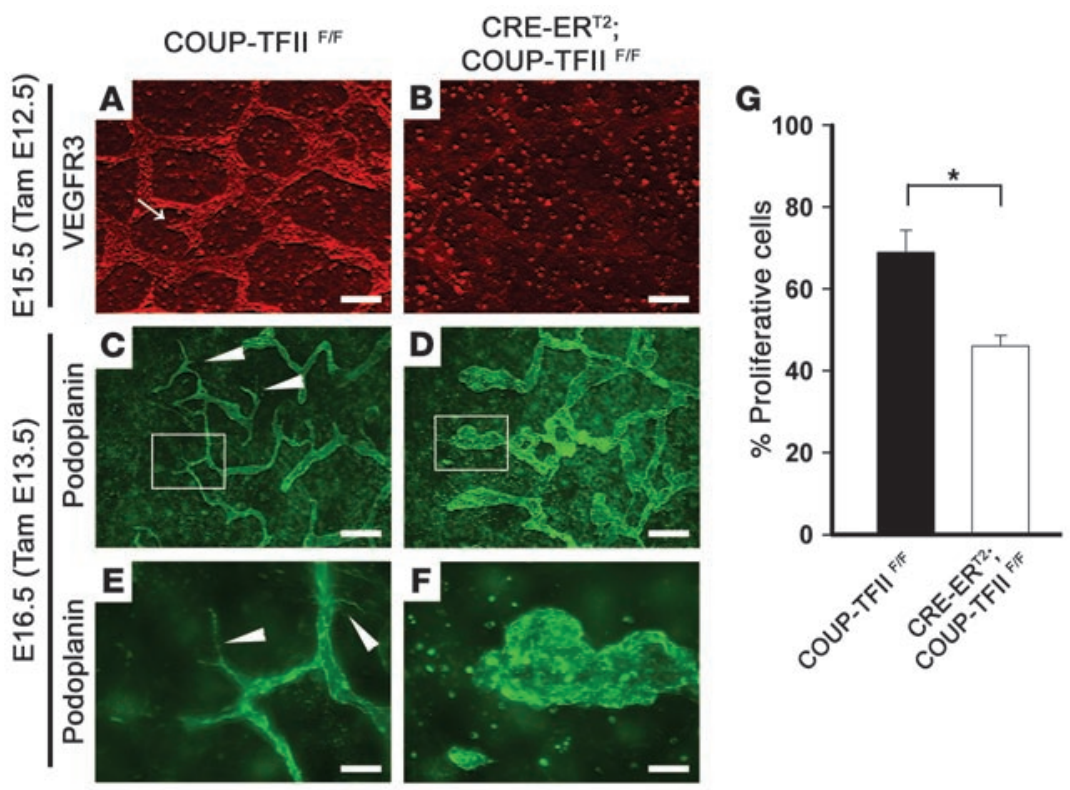

\section{Figure 4}

Defective sprouting lymphangiogenesis and reduced LEC proliferation in inducible COUP-TFII mutants. (A-F) Whole-mount preparation of back skin from COUP-TFIIF/F controls and CRE-ER ${ }^{T 2}$;COUP-TFIIF/F mutant embryos at E15.5 (Tam administration at E12.5) (A and B) and E16.5 (Tam administration at E13.5) (C-F), immunostained with VEGFR3 (red) and podoplanin (green), respectively. (A and B) COUP-TFIl mutants have impaired VEGFR3 production versus controls at E15.5. (C-F) Podoplanin immunostaining shows defective lymphatic sprouting and dilated cutaneous lymphatic vessels in mutants. ( $E$ and $F$ ) Higher-magnification images of the boxed regions in $\mathbf{C}$ and $\mathbf{D}$. The arrow in $\mathbf{A}$ and the arrowheads in $\mathbf{C}$ and $\mathbf{E}$ indicate lymphatic sprouts. Scale bars: $50 \mu \mathrm{m}$ (A, B, E, and F); $200 \mu \mathrm{m}$ (C and D). (G) COUP-TFIl mutants exhibited a reduction of LEC proliferation in the skin, as judged by the number of Ki67 and VEGFR3 double-positive cells. Error bars indicate SD. ${ }^{*} P<0.05$. of Prox1 in E11.5 conditional COUP-TFII mutants is LEC selective. Collectively, these findings suggest that the formation and differentiation of LECs was severely compromised in the COUPTFII mutant embryos.

Normally, from E9.5 onward, COUP-TFII proteins are detected in the venous endothelium (22). Venous marker EphB4 and arterial marker Nrp1 are expressed in the endothelium of the cardinal veins and dorsal aorta, respectively (data not shown). Induced COUP-TFII excision at the onset of E9.5 resulted in the reduced expression of EphB4 (Figure 2, E and F) and ectopic expression of Nrp1 in the venous endothelium of E11.5 mutant embryos (Figure $2, \mathrm{G}$ and $\mathrm{H}$ ), consistent with our previous observation that vein endothelium acquire an arterial character in the absence of COUPTFII (22). These results extend our previous findings and indicate that venous identity is programmed at an early embryonic stage and this process is COUP-TFII-dependent.

Taken together, these data confirm the notion that venous identity is required for early embryonic lymphangiogenesis. However, whether COUP-TFII has a cell-autonomous function in LECs during lymphangiogenesis has yet to be defined.

COUP-TFII is required for the maintenance of lymphatic identity after initial lymphatic sac formation. At E10, the LEC progenitors bud off the cardinal veins and undergo proliferation and migration to form the first primordial jugular lymphatic sacs by E12.5, which is followed by centrifugal sprouting to form an organized lymphatic network. To determine whether COUP-TFII is required for the initial formation of jugular lymphatic sacs, Tam was administrated to pregnant dams at E11.5, after LEC progenitors have already sprouted out from the cardinal veins, and the embryos were isolated and analyzed at E14.5. Most of the blood was contained within thin-walled vessels that stained weakly for VEGFR2 and VEGFR3 (Supplemental Figure 6, A-F). The expression of other lymphatic markers, Nrp2 and LYVE1, was almost undetectable (Supplemental Figure 6, G-J), indicating the malformation of lymphatic sacs in COUP-TFII mutants.

Next, to investigate whether COUP-TFII is required for the maintenance of LEC identity during late embryonic development,
COUP-TFII was deleted subsequent to the formation of the initial lymphatic sacs. For this purpose, Tam was administered to pregnant females at E12.5, and embryos were analyzed at E15.5. As observed above, distinct lymphatic sacs were present in both control and mutants at E12.5 and E15.5 (Figure 3, A-D); however, the lymphatic sacs and surrounding lymphatic vessels of mutants at E15.5 were blood filled (Figure 3D). Lymphatic markers, VEGFR3, $\mathrm{Nrp} 2$, and LYVE1 were clearly seen in the jugular lymphatic sacs of E12.5 and E15.5 control embryos and in E12.5 CRE-ERT2;COUP$T F I I^{F / F}$ embryos without Tam administration (Figure 3, E-G and I-K, and data not shown). In contrast, the expression of VEGFR3, Nrp2, and LYVE1 was markedly reduced in the COUP-TFII-deficient embryos (Figure 3, H and L, and data not shown). Interestingly, the expression of Prox 1 was unchanged in the lymphatic sacs and lymphatic vessels of COUP-TFII mutants (Figure 3, M-R and $\mathrm{U}-\mathrm{X})$. Together, these results suggest that during a developmental window between E12.5 and E15.5, COUP-TFII regulates lymphatic identity independent of Prox1.

Since the mammalian lymphatic system is derived from veins, we asked whether loss of COUP-TFII results in the acquisition of BEC characteristics in mutant LECs. The lymphatic vessels of control COUP-TFII ${ }^{F / F}$ embryos expressed LEC markers, including Prox1, LYVE1, Nrp2, and VEGFR3, but few or no BEC markers, such as endoglin, laminin, collagen IV, and CD34 (Figure 3, Q, S, U, and W, and Supplemental Figure 7, A, C, E, G, and I). In contrast, a marked reduction of the expression of LEC markers, such as LYVE1, Nrp2, and VEGFR3, and ectopic expression of BEC markers, endoglin, laminin, collagen IV, and CD34, was observed in the lymphatic vessels of E15.5 COUP-TFII mutant embryos (Figure 3, R, T, V, and X, and Supplemental Figure 7, B, D, F, H, and J). These results suggest that loss of COUP-TFII caused a reprogramming of LECs into BECs.

COUP-TFII is not only highly expressed in LECs but also expressed in VSMCs and pericytes. To investigate the possibility that COUP-TFII in VSMCs and/or pericytes may contribute to the lymphatic phenotypes displayed by the CRE-ER ${ }^{T 2}$;COUP-TFII ${ }^{F / F}$ mutants, we deleted COUP-TFII using the SM22 $\alpha$ Cre transgene 

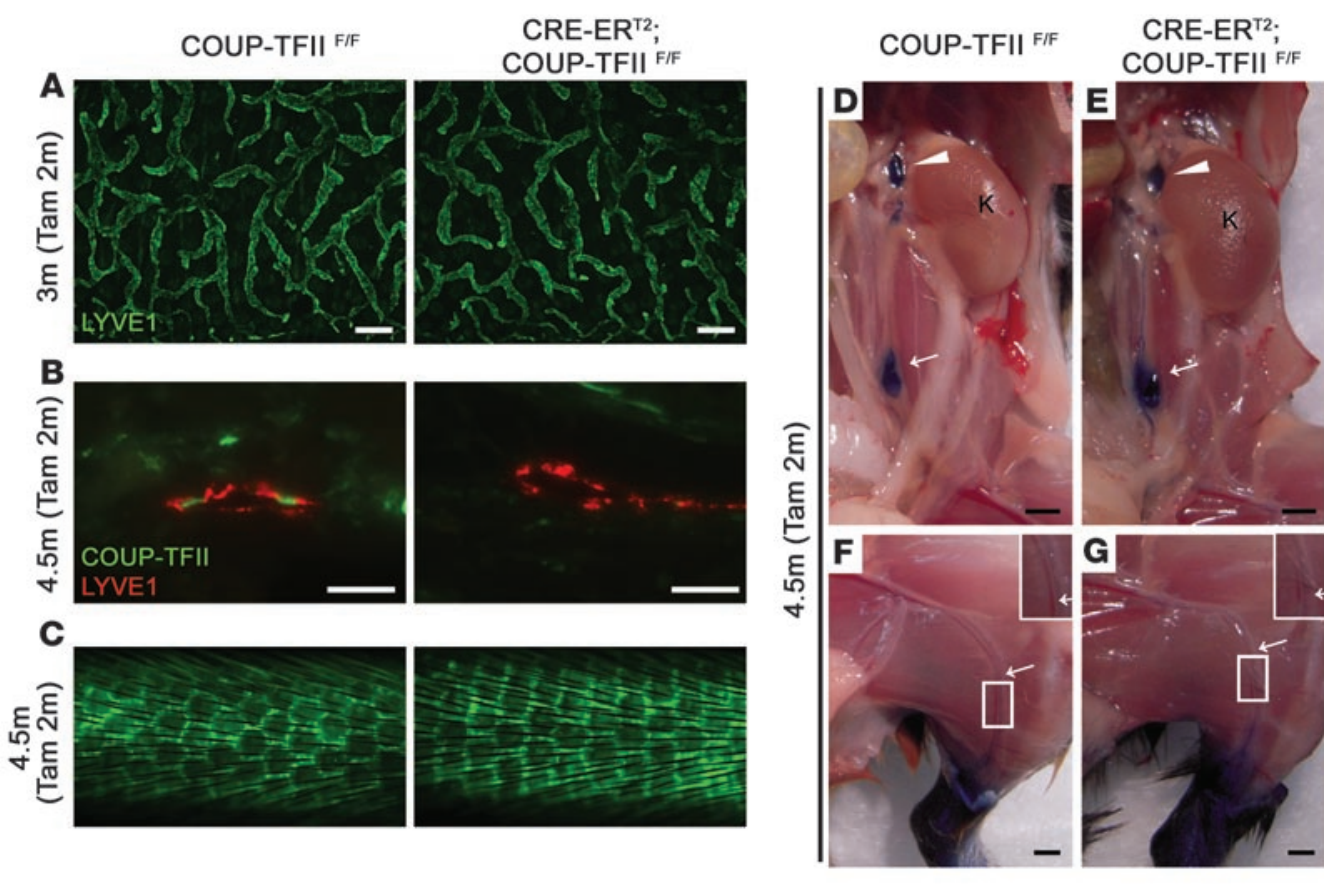
COUP-TFII FIF

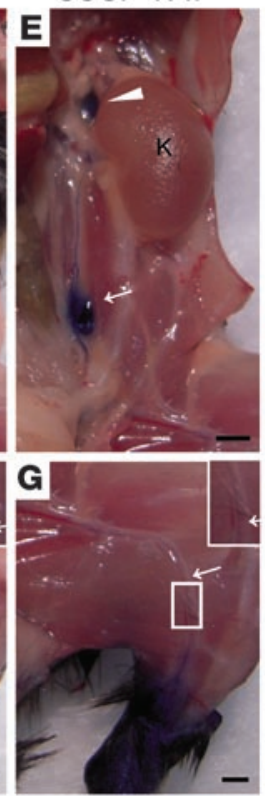

PyMT/+; F/F
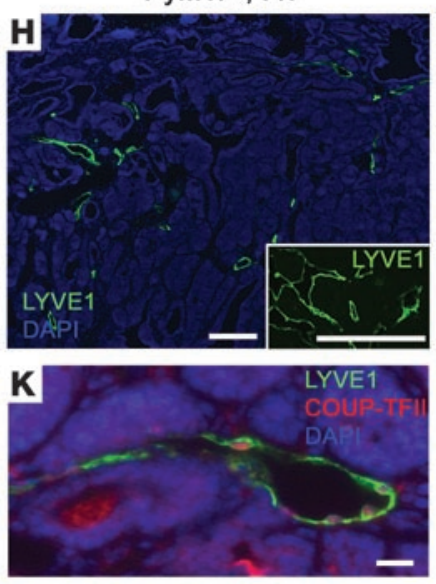

PyMT/+; CRE-ERT2/+; F/F
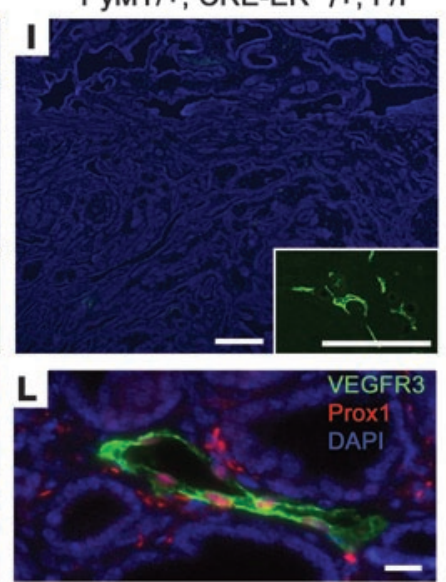

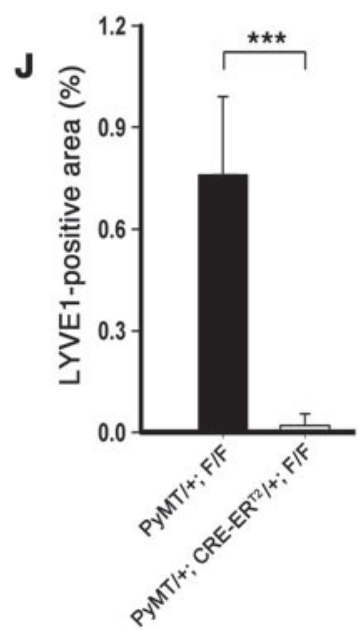

Figure 5

Inducible inactivation of COUP-TFII in adult mice, with Tam treatment at 2 months, does not affect normal function of lymphatic vessels but suppresses lymphangiogenesis in an animal model of cancer. (A) LYVE1 staining (green) of whole-mount ear preparations of 3-month-old controls and mutants. (B) COUP-TFII (green) and LYVE1 (red) double immunofluorescence was performed on ear sections of 4.5-month-old controls and mutants. (C) Fluorescent dextran lymphangiography of the tail. Levels of FITC-dextran uptake are similar in tail lymphatic vessels of controls and mutants. (D-G) Lymphangiography performed by injection of Evans blue dye into the hind limb foot pad of 4.5-month-old controls and mutants. Dye perfused large lymphatic vessels of the legs (F and $\mathbf{G}$ ) and draining iliac (arrows) and renal (arrowheads) lymph nodes (D and $\mathbf{E}$ ) of the abdomen to a similar extent in controls mutants. Insets in $\mathbf{F}$ and $\mathbf{G}$ are higher-magnification images of the boxed regions. K, kidney. (H and I) Immunofluorescence microscopy of mammary gland tumor sections from PyMT/+;F/F control or PyMT/+;CRE-ER $R^{T 2 /+; F / F ~ m u t a n t ~ l i t t e r m a t e s ~ a t ~} 4.5$ months, using an antibody against LYVE1 (green). Insets in $\mathbf{H}$ and I show LYVE1 staining (green) in tissue sections from a lymph node adjacent to the tumor. Inactivation of COUP-TFII results in the reduced tumor lymphangiogenesis but does not affect the preexisting lymphatic vessels in lymph nodes. $n=3$ for each group. (J) Statistical summary of LYVE1-positive areas in mammary gland tumor sections. Error bars indicate SD. ${ }^{* * *} P<0.01$. (K and $\left.\mathbf{L}\right)$ Immunofluorescence microscopy reveals that COUP-TFII proteins (red, K) are detected in LYVE1- (green, K), VEGFR3- (green, L), and Prox1-expressing (red, L) tumor-associated lymphatics of control mice. Scale bars: $200 \mu \mathrm{m}(\mathbf{A}, \mathbf{H}$, and I); $20 \mu \mathrm{m}(\mathbf{B}, \mathbf{K}$, and $\mathbf{L}) ; 500 \mu \mathrm{m}(\mathbf{C}) ; 2 \mathrm{~mm}$ (D-G).
(Supplemental Figure 8) (27). $S M 22 \alpha$ is expressed as early as E8.5 in cardiomyocytes. At E12.5, COUP-TFII was efficiently deleted (Supplemental Figure $8, A$ and $B$ ) and $\beta$-gal was turned on in $\alpha$-SMApositive VSMCs of the carotid artery (Supplemental Figure $8, \mathrm{C}$ and D). Importantly, the expression of lymphatic markers, such as VEGFR3, Prox1, Nrp2, and LYVE1, was comparable between controls and SM22 $\alpha$ CreCOUP$T_{F I I}^{F / F}$ embryos (Supplemental Figure 8, A and B, and data not shown). Similarly, when COUP-TFII was inactivated in VSMCs at E14.5, VEGFR3 expression was maintained (Supplemental Figure 8, E-H). Collectively, these results indicate that COUP-TFII, in the VSMCs and/or pericytes, contributes little, if any, to the lymphatic defects seen in the CRE-ER ${ }^{T 2}$;COUP-TFII ${ }^{F / F}$ mutants. Instead, it is consistent with the notion that the overall phenotype displayed by the CRE-ER ${ }^{T 2}$;COUP-TFII ${ }^{F / F}$ embryos mainly derives from the loss of COUP-TFII function in the endothelium.

Together, these results demonstrate that COUP-TFII is required for the initiation as well as the maintenance of lymphatic cell identity prior to lymphatic maturation, and inducible ablation of COUP-TFII during embryonic stages (between E12.5 and E15.5) dedifferentiates Prox1expressing LECs into BECs.

COUP-TFII is required for the lymphangiogenic sprouting of the dermal lymphatic vasculature. The primitive lymphatic plexus develops prenatally and then undergoes remodeling or maturation to form a hierarchical lymphatic network. During the process of lymphatic vascular maturation, new capillaries arise by sprouting from the preexisting vasculature. To further 
A

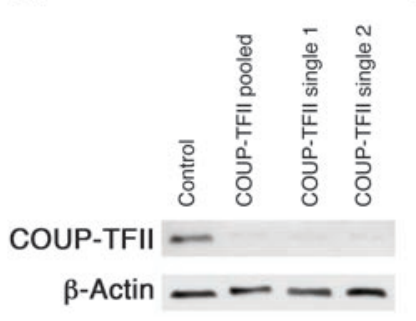

B

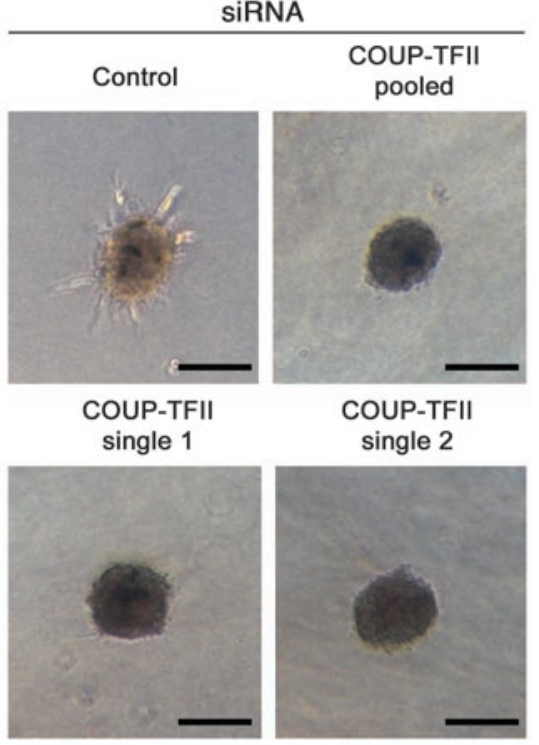

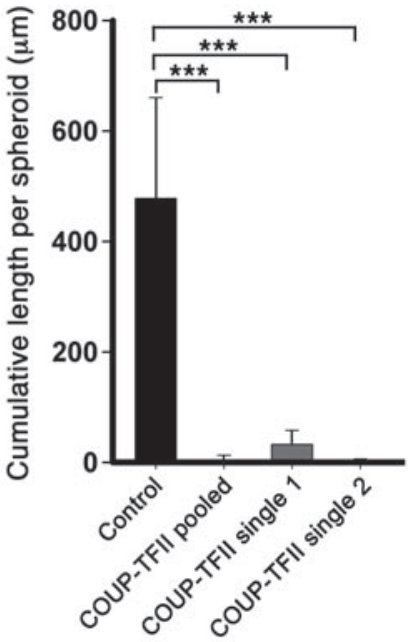

COUP-TFII pooled siRNA
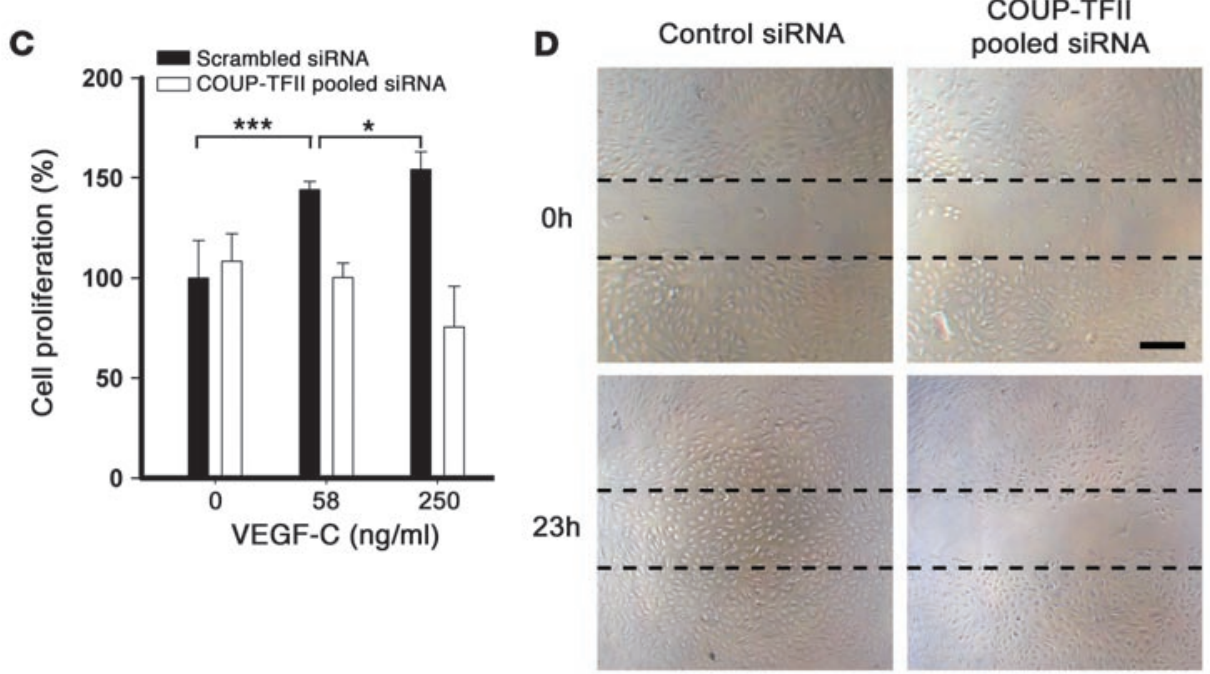

\section{Figure 6}

COUP-TFII knock down impairs in vitro lymphangiogenesis, VEGF-C-induced cell proliferation, and migration of LECs. (A) Human primary LECs were transfected with control or COUP-TFII-pooled or 2 different COUP-TFII siRNAs. Cell lysates were subjected to Western blotting with antibodies against COUP-TFII and $\beta$-actin. (B) Representative images and statistical summary of 3-dimensional in vitro lymphangiogenesis assays, with spheroids generated from control- or COUP-TFII-pooled siRNA- or 2 different COUP-TFII siRNA-treated LECs. The cumulative sprout length was quantified after 24 hours. Extensive endothelial outgrowth can be observed in control spheroids but not in COUP-TFII-silenced spheroids. Scale bars: $100 \mu \mathrm{m}$. Error bars indicate SD. ${ }^{* *} P<0.01$. (C) Cell proliferation assay measuring LEC response to increasing concentrations $(0,58,250 \mathrm{ng} / \mathrm{ml})$ of VEGF-C. Stimulation of scrambled siRNA-transfected but not COUP-TFII-pooled siRNA-transfected LECs induces a dose-dependent increase in cell proliferation. Error bars indicate SD. ${ }^{*} P<0.05$, ${ }^{\star * *} P<0.01$. (D) Wound scratch assay of LEC response to 250 $\mathrm{ng} / \mathrm{ml}$ VEGF-C. The edges of the wound scratch are shown by dashed lines.

assess the role of COUP-TFII in lymphangiogenesis, we induced COUP-TFII ablation at E12.5 or E13.5 and analyzed lymphatic vessel sprouting using embryonic back skin as a model. As shown by whole-mount VEGFR3 immunostaining, the dermal lymphatic capillaries of E15.5 control embryos formed a primitive lymphatic plexus with few sprouts and expressed high levels of VEGFR3 (Figure 4A), while VEGFR3 expression was significantly reduced in the dermal lymphatic capillaries of E15.5 mutant embryos (Figure 4B). More sprouts emerged from the initial lymphatic capillaries at E16.5, as judged by whole-mount podoplanin immunostaining
(Figure 4, C and E, arrowheads). In contrast, numerous lymphatic capillaries in COUP-TFII mutants were irregular and grossly dilated (Figure 4, D and F). Strikingly, they failed to form filopodial extensions from the vessels, which resulted in blunt-ended protrusions (Figure 4, D and F). These data suggest that the sprouting of lymphatic capillaries is disrupted in the absence of COUP-TFII. Therefore, COUP-TFII is required for lymphangiogenic sprouting.

To determine whether dermal lymphatic hypoplasia in CRE$E R^{T 2}$;COUP-TFII ${ }^{F / F}$ mutants could be partially caused by defective LEC proliferation, we performed Ki67 immunostaining to measure 

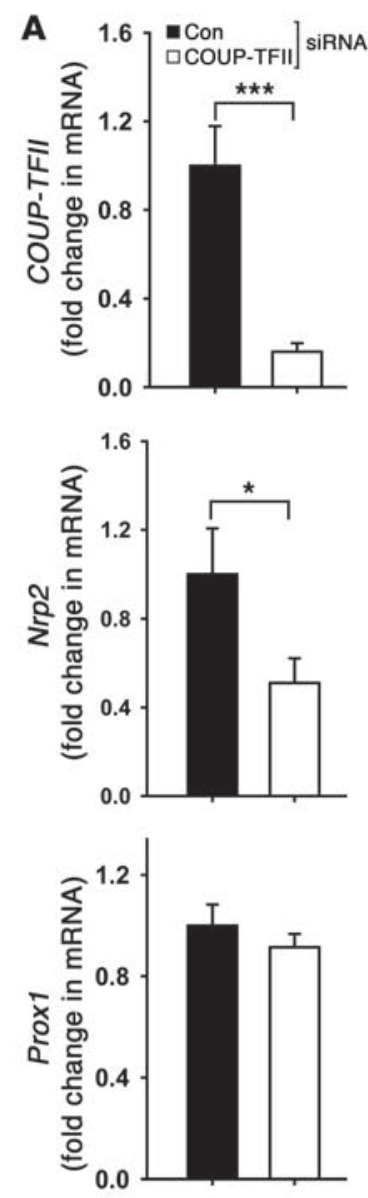

$\mathbf{F}$

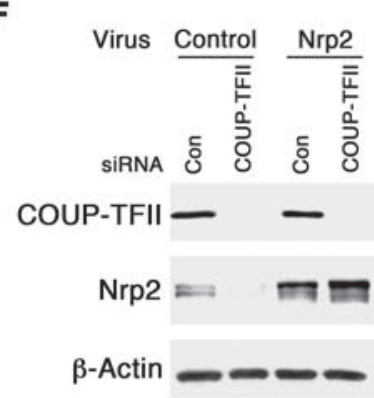

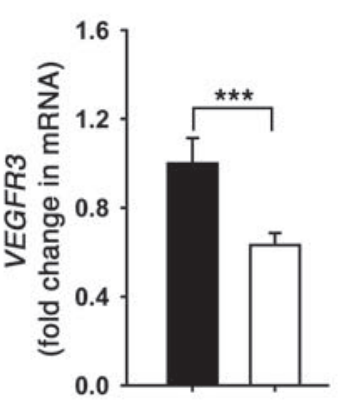
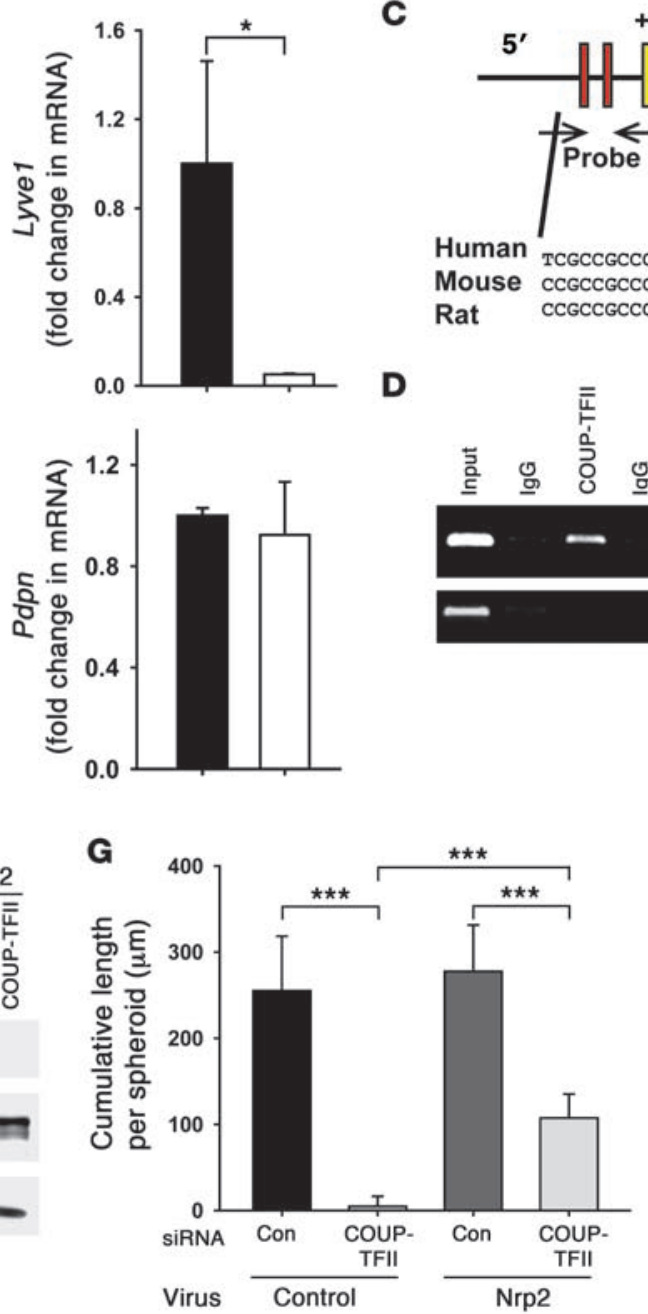

B
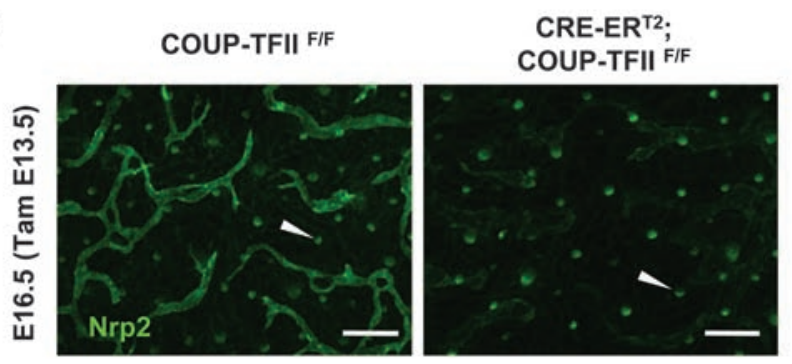

C

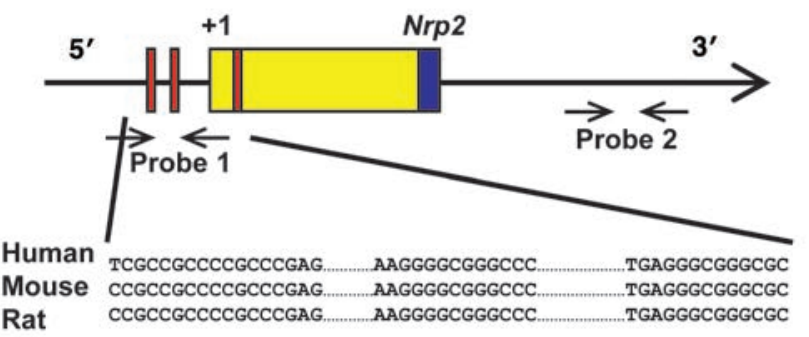

E
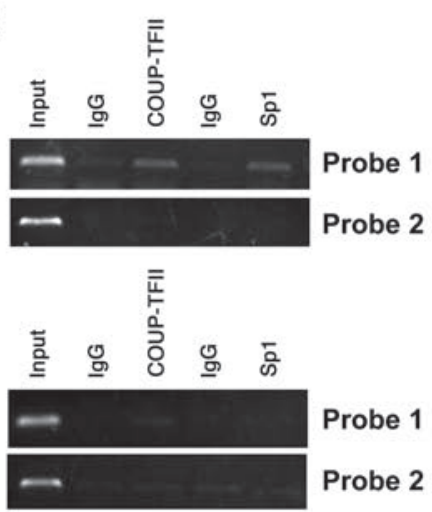

Figure 7

COUP-TFII mediates lymphangiogenic responses by directly regulating Nrp2 expression. (A) Quantitative RT-PCR analysis of lymphatic-specific transcripts in RNA isolated from COUP-TFIl or scrambled siRNA-transfected human primary LECs (normalization to 18S rRNA). Error bars indicate SD. ${ }^{\star} P<0.05,{ }^{* \star *} P<0.01$. (B) Whole-mount staining for Nrp2 in embryonic back skin of COUP-TFIIF/F control and CRE-ER ${ }^{T 2}$;COUPTFIFIF mutant embryos at E16.5 (Tam administration at E13.5). Nrp2 expression in lymphatic vessels but not in hair follicles (arrowheads) is markedly reduced in inducible COUP-TFII mutants compared with controls. Scale bar: $200 \mu \mathrm{m}$. (C) Sequence alignment of putative Sp1-binding sites within Nrp2 5' untranslated regions from different species. The red boxes indicate the Sp1-binding site, the yellow box indicates the $5^{\prime}$ untranslated region, and the blue box indicates the translated portion of exon. (D) ChIP analysis of COUP-TFII and Sp1 binding to the putative Sp1-binding sites of human Nrp2 promoter in LECs. (E) ChIP analysis of COUP-TFII and Sp1 binding to the conserved Sp1-binding sites of human Nrp2 promoter in control siRNA-treated LECs (top panel) and in Sp1-specific siRNA-treated LECs (bottom panel). (F) Detection of Nrp2, COUP-TFII, and $\beta$-actin by Western blot of human primary LECs transfected with COUP-TFII-specific or scrambled siRNA or transduced with lentivirus expressing full-length human Nrp2 or GFP (control). (G) Statistical summary of 3-dimensional in vitro lymphangiogenesis assays, with spheroids generated from human primary LECs transfected with COUP-TFII-specific or control siRNA or transduced with a control GFP lentivirus or one expressing full-length human Nrp2. Spheroids generated from overexpression of Nrp2 in COUP-TFII-silenced LECs have higher lymphangiogenic activity than COUP-TFII-silenced spheroids. Error bars indicate SD. ${ }^{* \star} P<0.01$. 
the proliferation rate of dermal LECs in E15.5 conditional COUPTFII mutants (with Tam administration at E12.5). The VEGFR3 and Ki67 double-positive cells, indicative of proliferating LECs, were counted, and the quantification result was presented in Figure 4G. The difference in the number of Ki67-positive cells between controls and mutants was evident (Figure 4G). Taken together, COUP-TFII is required for lymphangiogenic sprouting and normal LEC proliferation during the late developmental stages.

COUP-TFII is not required for the maintenance of quiescent lymphatic vessels in the adult. As shown above, COUP-TFII is required for the establishment of lymphatic identity during embryogenesis. However, it is unclear whether COUP-TFII is required for lymphatic vessel maintenance subsequent to maturation of lymphatic vessels in the adult. To this end, we injected $0.5 \mathrm{mg}$ Tam per day for 5 consecutive days to 2 month-old COUP-TFII ${ }^{F / F}$ and CRE-ER ${ }^{T 2}$;COUP-TFII $I^{F / F}$ mice to delete COUP-TFII. One month after Tam administration, mouse ears were examined by whole-mount immunostaining with antibodies against the lymphatic markers LYVE1 and podoplanin. As shown in Figure 5A and Supplemental Figure 9, the lymphatic vessels in the mouse ear formed networks of small and often blindended capillaries in both control and COUP-TFII mutant mice. In addition, the expression levels of LYVE1 and podoplanin in the lymphatic vessels of CRE-ER $R^{T 2}$;COUP-TFII ${ }^{F / F}$ mice were indistinguishable from those in the COUP-TFII ${ }^{F / F}$ mice, suggesting that the adult lymphatic identity was maintained in the absence of COUPTFII (Figure 5A and Supplemental Figure 9).

Next, to confirm that the unchanged lymphatic cell identity is not due to the inefficient excision of COUP-TFII in adult quiescent lymphatic vasculature, antibodies against COUP-TFII were used for immunofluorescence microscopy. COUP-TFII was efficiently deleted in adult mouse lymphatic vessels, as determined by the expression of lymphatic markers LYVE1, podoplanin, and Prox1 (Figure 5B and data not shown). To investigate the specific efficiency of the inactivation of COUP-TFII in ECs, we isolated ECs from the adult lungs using FACS and then performed allele excision analysis and measured COUP-TFII mRNA expression. Both results showed that COUP-TFII was efficiently deleted in the adult lung ECs (Supplemental Figure 10, A-C). Immunoblotting cell lysates from the lung further revealed that COUP-TFII was efficiently deleted when CRE-ER ${ }^{T 2}$;COUP-TFII ${ }^{F / F}$ mice were treated with Tam but not with oil (Supplemental Figure 10D). Consistent with the normal patterning of lymphatic vasculature in CRE-ER ${ }^{T 2}$;COUP-TFII ${ }^{F / F}$ mice, no obvious signs of lymphedema or chylous fluid accumulation were observed in the mutant animals (data not shown).

An unanswered question is whether the apparent normal-looking lymphatic vessels present in COUP-TFII mutant mice are functionally intact. To assess this, lymphatic vessel function was evaluated by visualizing the uptake and drainage of large-molecular-weight fluorescent FITC-dextran or Evans blue dye after subcutaneous injection into the mouse tail or foot pad. As shown in Figure 5C, the dextran was taken up by the hexagonal lymphatic network of the mouse tail in both control and COUP-TFII mutant mice. Similar results were observed with the intradermal injection of FITC-dextran into the mouse ear (data not shown). Consistently, the Evans blue dye injected into the hind limb foot pad was rapidly drained into lymphatic vessels and the draining lymphatic nodes (Figure 5, D-G, arrows and arrowheads) in both control and mutant mice, indicating that COUP-TFII is dispensable for the fluid drainage function of fully mature adult lymphatics. Together, these data suggest that under the conditions used here, inactivation of COUP-TFII does not affect the maintenance or functions of quiescent adult lymphatics.

Inactivation of COUP-TFII results in a reduction of tumor lymphatics. The lymphatic vessels are in a quiescent state in the adult. However, under pathological conditions, such as tumorigenesis, neolymphangiogenesis becomes very apparent. To examine whether COUP-TFII plays a role in neo-lymphangiogenesis, we used a well-studied spontaneous, mammary gland tumor mouse model, the MMTV-polyoma middle T antigen (MMTV-PyMT) mouse, for our study. To induce mammary tumorigenesis in COUP-TFII mutant mice, MMTV-PyMT mice were crossed to COUP-TFII ${ }^{F / F}$ and CRE-ER ${ }^{T 2}$;COUP-TFII ${ }^{F / F}$ mice. The resulting MMTV-PyMT/+;CRE$E R^{T 2}$;COUP-TFII ${ }^{F / F}$ mice (hereafter referred to as $P y M T /+$;CRE-ER ${ }^{T 2} /+$; $F / F$ mice) were phenotypically indistinguishable from control MMTV-PyMT/+;COUP-TFII ${ }^{F / F}$ mice (hereafter referred to as PyMT/+; $F / F$ mice) in the absence of Tam treatment (data not shown).

The PyMT mouse model develops aggressive polyclonal tumors that fill the entire mammary fat pad by as early as 7-8 weeks, and a large percentage of mice develop carcinoma at around 14 weeks (28). We used this mouse model to examine the mammary fat pad 14 weeks after Tam administration. As judged by immunofluorescence staining for the lymphatic marker LYVE1, LYVE1-expressing lymphatic vessels were easily seen within the carcinomas of the PyMT/+;F/F control mice (Figure $5 \mathrm{H}$ ) but not in the mice lacking COUP-TFII (Figure 5I). Quantification further confirmed a significant difference in the LYVE1-positive areas in the tumors of the control versus mutant mice (Figure 5J). Interestingly, LYVE1 signals in the preexisting lymphatic vessels in lymph nodes adjacent to the tumor remained the same (Figure 5, $\mathrm{H}$ and I, insets), suggesting that only the newly formed lymphatic vessels in the tumor but not the preexisting vessels were affected in COUP-TFII-deficient mice. The expression of VEGFR3 and Nrp2 in tumor lymphatics was decreased in COUP-TFII mutant mice (Supplemental Figure 11). To confirm that COUP-TFII is expressed in the tumor-associated lymphatics, a COUP-TFII antibody was used to stain mouse tumor tissues of control mice. Indeed, tumor lymphatics expressed COUP-TFII in addition to LYVE1, Prox1, and VEGFR3 (Figure 5, K and L). Taken together, these data suggest that COUP-TFII is involved in neo-lymphangiogenesis in the adult under non-homeostatic conditions and that targeted deletion of COUP-TFII inhibits tumor lymphangiogenesis.

Silencing of COUP-TFII abolishes lymphatic sprout formation in vitro. VEGF-C, a lymphangiogenic factor, is essential for the initial sprouting, the subsequent survival, and the migration of LECs (11). We therefore investigated whether downregulation of COUPTFII would affect any of these cellular responses in vitro. Human primary LECs expressing COUP-TFII (29) were transfected with 2 different COUP-TFII-targeting short, interfering RNAs (siRNAs) or control siRNA. COUP-TFII protein in LECs was downregulated efficiently 48 hours after transfection (Figure 6A). To test whether COUP-TFII is involved in VEGF-C-induced lymphangiogenic activity of LECs, we examined in vitro angiogenesis in a 3-dimensional spheroidal assay. Control, 2 different COUP-TFII siRNAs, or COUPTFII-pooled siRNA-transfected LEC spheroids, with defined size and cell number, were embedded in collagen gels and then treated with VEGF-C for 24 hours. Outgrowth of capillary-like structures was assessed. As shown in Figure 6B, control siRNA-treated LEC spheroids elicited numerous spontaneous sprouts, while silencing of COUP-TFII in LEC spheroids almost completely inhibited the 
formation of lymphatic endothelial sprouts in vitro. These results suggest that the lack of tumor lymphatic vessel formation in vivo (Figure 5, H and I) may be due to impaired sprouting of lymphatic vessels. These results indicate that COUP-TFII is required for lymphangiogenesis in a cell-autonomous manner.

Sprouting of LECs is a multistep process, involving both proliferation and migration. To address whether COUP-TFII is important for VEGF-C-induced proliferation and/or migration of LECs, COUP-TFII-pooled siRNA was used to silence the expression of COUP-TFII in LECs. Cellular proliferation was enhanced in a dosage-dependent manner in response to VEGF-C in control siRNA-treated cells, while VEGF-C-induced proliferation was lost in COUP-TFII knockdown cells (Figure 6C and Supplemental Figure 12). Moreover, in a wound healing assay, migration of LECs in response to VEGF-C was impaired when COUP-TFII was knocked down in comparison with that of the control (Figure 6D), which is consistent with previous results using VEGF-C as an attractant in a chamber migration assay (30). Taken together, these results demonstrate that COUP-TFII is required for VEGF-C-induced lymphangiogenesis in cultured human primary LECs.

Altered gene expression in COUP-TFII knockdown LECs. To identify molecular mechanisms underlying the COUP-TFII-dependent regulation of lymphangiogenesis, we used a candidate gene approach to compare expression profiles of control and COUPTFII knockdown human primary LECs. Consistent with the in vivo results, silencing of COUP-TFII expression in LECs led to a significant downregulation of lymphatic marker genes, including VEGFR3, Nrp2, and Lyve1 (Figure 7A), suggesting that COUP-TFII acts upstream of VEGFR3, Nrp2, and LYVE1. However, the expression levels of Prox 1 and podoplanin $(P d p n)$ were not affected (Figure $7 \mathrm{~A})$, in agreement with previous observations by others (29, $30)$. Given that the COUP-TFII level remains the same while Pdpn expression decreases in Prox 1 knockdown LECs (29), these data imply that COUP-TFII likely functions parallel to Prox1 to mediate lymphangiogenesis. We suggest that LECs lose certain features of their lymphatic identity with the reduction of COUP-TFII.

Direct regulation of Nrp2 transcription by COUP-TFII in LECs. As mentioned above, lymphangiogenesis is governed by multiple distinct factors, and $\mathrm{Nrp} 2$ is one of these important factors. Nrp2 has been shown to be a positive modulator of developmental lymphangiogenesis, and its activity is required for VEGF-C-dependent migration and survival of cultured ECs $(16,18)$. Since Nrp2 is downregulated in COUP-TFII knockdown cells and in COUP-TFII mutants (Figure 7A and Figure 3, K and L), we hypothesized that Nrp2 might mediate COUP-TFII function to regulate lymphangiogenesis. To address this issue, we first asked whether COUP-TFII regulates Nrp2 expression in the embryonic back skin lymphatic vasculature. Whole-mount immunofluorescence staining showed that a marked reduction of $\mathrm{Nrp} 2$ expression was observed in the dermal lymphatic vasculature of COUP-TFII-deficient mice in comparison with the control mice at E16.5 (Figure 7B). Moreover, Nrp2 protein expression was abolished in the cardinal veins of E9.5 COUP-TFII-null embryos in comparison with the wild-type embryos (Supplemental Figure 13). These data indicate that Nrp2 is a physiologically relevant COUP-TFII downstream target gene in vivo.

To determine whether Nrp2 is a direct downstream target of COUP-TFII, we carried out ChIP assays. We and others have previously demonstrated that when COUP-TFs act as a positive regulator to enhance its target gene expression, COUP-TFs interact with $\mathrm{Sp} 1$ on Sp1-binding sites to positively regulate their target genes. Mutations of Sp1-binding sites abolish COUP-TFII-dependent enhancement of NGFI-A promoter activity (31). Similarly, knockdown of Sp1, using Sp1-specific siRNA, abolishes COUPTFII-dependent regulation of IGF1 expression (32). These results indicate that positive regulation of target gene expression by COUP-TFII requires Sp1. To assess whether COUP-TFII regulates Nrp2 expression through interactions with Sp1, we first searched for potentially conserved Sp1-binding sites in the human, mouse, and rat sequences and found at least 3 conserved sites around the transcription start site of the Nrp2 promoter (Figure 7C, red boxes). We then performed ChIP assays, using human primary LECs as a source of material, to assess whether endogenous COUP-TFII is recruited to the Nrp2 promoter to modulate Nrp2 expression. COUP-TFII was found to be preferentially recruited to the promoter regions containing Sp1-binding sites, while COUP-TFII is not recruited to the $3^{\prime}$ region lacking $S p 1$-binding sites (Figure $\left.7 D\right)$. In parallel, Sp1 was also recruited preferentially to the same region containing the Sp1-binding sites as COUP-TFII but not to the region lacking $\mathrm{Sp}$ 1-binding sites. To substantiate that the recruitment of COUP-TFII to the Nrp2 promoter requires the presence of Sp1, and $S p 1$ was knocked down in LECs. Indeed, the recruitment of COUP-TFII and Sp1 to the Nrp2 promoter was significantly reduced in the Sp1-depleted cells (Figure 7E, bottom panel) as compared with the control LECs (Figure 7E, top panel). To further test whether COUP-TFII binding to the Nrp2 promoter leads to activation of transcription, we performed luciferase reporter assays using a 3.9-kb mouse $N r p 2$ promoter fragment that included the 3 conserved Sp1-binding sites. We transiently transfected a luciferase reporter containing the Nrp2 promoter (pGL2-Nrp2) or a control vector (pGL2 basic) in COUP-TFII-overexpressing and control HEK293T cells. Levels of relative luciferase activity induced by the Nrp2 promoter reporter were significantly increased in COUPTFII-transduced cells compared with control cells (Supplemental Figure 14). Collectively, these results strengthened our model that the recruitment of COUP-TFII to the Nrp2 promoter is Sp1 dependent, and COUP-TFII activated Nrp2 transcription.

Nrp2 overexpression partially rescues the impaired lymphangiogenic sprouting in COUP-TFII knockdown LECs. If $\mathrm{Nrp} 2$ indeed mediates COUP-TFII function to induce lymphangiogenesis in COUP-TFII knockdown LECs, our prediction was that ectopic expression of Nrp2 in LECs might rescue sprouting lymphangiogenesis defects. To test this hypothesis, LECs were transfected with control or COUP-TFII-pooled siRNA, and cells were then infected with Nrp2 or control GFP-expressing lentiviruses. Overexpression of $\mathrm{Nrp} 2$ in LECs had no influence on COUP-TFII levels (Figure 7F). Consistent with the above results, silencing of COUP-TFII gene expression decreased the lymphangiogenic responses of LECs (Figure 7G). More importantly, lentiviral overexpression of Nrp2 in COUP-TFII knockdown LECs partially rescued the VEGF-C-induced lymphangiogenic activity (Figure $7 \mathrm{G}$ ), suggesting that COUP-TFII mediates lymphangiogenesis, at least in part, by modulating the expression of $\mathrm{Nrp2}$.

\section{Discussion}

Our results reveal a central role of COUP-TFII in the determination of lymphatic cell fate and the maintenance of LEC identity in development. Conditional ablation of COUP-TFII in mice results in the malformation of primitive lymphatic vessels, lymphatic sacs, and impairment of sprouting lymphangiogenesis. Exploring the underlying mechanism, we show that COUP-TFII con- 
trols sprouting lymphangiogenesis through direct transcriptional regulation of $\mathrm{Nrp2}$, the coreceptor for VEGF-C in LECs. Finally, our results suggest that COUP-TFII is also an essential factor for tumor-induced neo-lymphangiogenesis in a spontaneous mouse breast cancer model. Thus, COUP-TFII modulates developmental as well as postnatal pathological lymphangiogenesis.

Partial rescue of the COUP-TFII phenotype by Nrp2 expression indicates that other molecules may also be involved. The ROSA26CRE-ERT2 mouse strain also efficiently inactivates COUPTFII in the mesenchymal tissues and VSMCs. Although the lack of phenotypes in the smooth muscle-specific COUP-TFII-deficient mice largely excluded the contribution of COUP-TFII in VSMCs, with respect to the overall phenotypes displayed by the CRE$E R^{T 2}$;COUP-TFII ${ }^{F / F}$ mutants, we could not rule out the contribution of cells other than ECs and smooth muscle cells/pericytes. For instance, COUP-TFII is expressed in the mesenchymal tissues surrounding the dorsal aorta and cardinal vein where angiopoietin 1 (Ang1) is highly expressed. Angiopoietin family members, Ang1 and Ang2, have been shown to regulate angiogenesis and lymphangiogenesis through the Tie 1 and/or Tie 2 receptor tyrosine kinase that is expressed both in BECs and LECs (33). Ectopic expression of Ang1 promotes lymphatic sprouting and lymphangiogenesis in vitro and in vivo $(34,35)$. Additionally, Ang2-deficient mice displayed disorganized and hypoplastic dermal lymphatic capillaries, and the lymphatic defects can be rescued by Ang1 (36). These studies suggest that Ang1 and Ang2 share redundant functions in lymphatic vessels and the angiopoietin-Tie system is important for lymphangiogenesis. Since Ang1 expression is markedly reduced in COUP-TFII-null embryos (23), it is possible that the reduction of Ang-1 expression may contribute to the defects displayed by the COUP-TFII mutants. A successful ablation of COUP-TFII exclusively in LECs may help address these questions.

Another surprising finding that emerged from our studies was that LECs ectopically expressed BEC markers, even though Prox1 expression was not affected. The independence of Prox1 on LEC specification in COUP-TFII mutants was particularly intriguing considering that LECs could be partially reprogrammed into BECs in the presence of Prox1. Our current study and those of others $(29,30)$ suggest that COUP-TFII acts jointly with Prox1 in the pathway of LEC specification, since downregulation of either factor is able to suppress LEC fate and induce BEC characteristics. The idea that COUP-TFII and Prox1 proteins interact to specify LEC identity is also supported by the knockdown of Prox 1 or/and COUP-TFII in cultured LECs. The expression of the lymphatic markers Vegfr3 and Fgfr3 was synergistically downregulated in COUP-TFII and Prox 1 double-knockdown LECs. In these doubleknockdown cells, intercellular adhesion molecular 1 (ICAM-1) and monocyte chemotactic protein-1 (MCP-1), which are more abundantly expressed in BECs than in LECs, were cooperatively upregulated (29). Taken together, our findings support the notion that LEC identity is reversible during development. We also provide what we believe to be an important new role for COUP-TFII in lymphatic cell fate decision.

Given the facts that COUP-TFII and Prox1 share similar functions on lymphatic specification, and that they physically interact with each other in vitro $(29,30)$, we asked whether COUP-TFII functions independently or in cooperation with Prox1 to regulate the establishment and the maintenance of lymphatic identity. Our data and those of Lee et al. showed that Pdpn, a lymphatic marker, was mainly regulated by Prox 1 , since its expression decreased when Prox 1 was downregulated but remained unchanged in the COUP-TFII-depleted cells (Supplemental Figure 15A) (29). In contrast, the expression of lymphatic markers, LYVE1 and Nrp2, depends on COUP-TFII but not on Prox1 (Supplemental Figure 15A) (29). Interestingly, when we analyzed the promoter of the FGFR3 gene, whose transcriptional activity was shown to be synergistically activated by both COUP-TFII and Prox1 (29), we found that both COUP-TFII and Prox1 were directly recruited to the region containing putative Prox1-binding sites on the FGFR3 promoter (Supplemental Figure 15, B and C). Intriguingly, knockdown of Prox1 did not affect the recruitment of COUP-TFII on the FGFR3 promoter by ChIP assay (Supplemental Figure 15D). Therefore, COUP-TFII binding to the FGFR3 promoter does not require Prox1, which may indicate that they bind to 2 different sites. Indeed, there are Sp1 sites in the region in which COUP-TFII was recruited. Our results are also consistent with results obtained from transient transfection assays using a 3-kb FGFR3 promoterdriven luciferase reporter, in which the ability of COUP-TFII to activate the FGFR3 promoter activity is not affected by a DNAbinding defective mutant of Prox1, while the synergistic activation of the reporter by COUP-TFII and Prox1 is compromised (29). Therefore, activation of the FGFR 3 promoter requires both COUPTFII and Prox1. These findings suggest that the recruitment of COUP-TFII to the FGFR3 promoter is Prox 1 independent, but the synergistic activation requires binding of both Prox 1 and COUPTFII to the promoter. Taken together, these findings suggest that COUP-TFII and Prox1 may act independently or cooperatively to regulate their downstream targets, depending on the promoter and cellular contexts. However, we can not rule out the possibility that the requirement for COUP-TFII and/or Prox1 may differ due to heterogeneity of the LECs, the developmental stage of LECs, and the type of lymphatic vessels.

Another issue is whether COUP-TFII regulates Prox1 transcription in vivo. Deletion of COUP-TFII before E9.5 results in the absence of Prox1-positive lymphatic endothelial progenitor cells (Figure 2 and Supplemental Figure 4). Deletion of COUP-TFII at E10.5 results in the reduction of Prox1 expression in the malformed jugular lymphatic sacs (Supplemental Figure 16). These results indicate that the induction of Prox1 expression in LECs prior to or at E10.5 is likely COUP-TFII dependent. The reduced Prox1 expression could arise from the loss of venous endothelial and/or lymphatic endothelial progenitor cell identity or from defects in differentiation of LECs, depending on the window of development. Furthermore, we showed a window during development (E12.5-E15.5) in which the maintenance of Prox1 expression is COUP-TFII independent. Therefore, it is unlikely that Prox 1 expression is directly regulated by COUP-TFII. This result is consistent with the findings of Lee et al. and Yamazaki et al. (29, 30), showing that Prox1 expression is not regulated by COUP-TFII. Collectively, our results indicate that lymphatic formation and differentiation are compromised in the absence of COUP-TFII.

Despite the persistent expression of COUP-TFII in the adult mature lymphatic vessels, normal lymphatic function remains intact when COUP-TFII is inactivated in adult mice. Similar to our findings, blocking of VEGFR3 signaling in adult mice by means of recombinant VEGFR3-Ig fusion protein has little effect on mature lymphatic vessels (15). Furthermore, an antibody to Nrp2 that blocks VEGF-C binding does not affect established lymphatics in normal adult mice (18). Together, these results suggest that disruption of VEGFC/Nrp2/VEGFR3 signaling, either via COUP- 
TFII or direct inhibition, hampers early lymphatic vessel development but has little impact on the quiescent lymphatic vessel function in the adult.

Most interestingly, we found that COUP-TFII is essential for neo-lymphangiogenesis in adult mice. Lymphatic vessels, like the blood vessel system, are quiescent in most adult tissues under homeostatic conditions. In adults, lymphangiogenesis takes place only in certain pathological conditions, such as during tumorigenesis, inflammation, and trauma induced by radiation treatment or surgery (1). Recently, tumor lymphangiogenesis has received considerable attention due to the abundant clinical evidence indicating that metastasis of malignant tumors via lymphatic vessels to regional lymph nodes is an early sign of cancer spread in patients, and it occurs as frequently as hematogenous metastasis. Here, we showed that COUP-TFII is a crucial regulator for lymphangiogenesis, not only in embryonic development, but also in adults. Disruption of COUP-TFII activity in a mammary gland tumor mouse model substantially reduces neo-lymphangiogenesis. Consistent with this idea, tumor lymphangiogenesis and lymphatic metastasis can be efficiently inhibited by soluble VEGFR3 fusion proteins acting as a VEGF-C/-D trap (20,37-40), by antibodies neutralizing VEGFR3 (19, 41-43), and by small molecular VEGFR2/VEGFR3 kinase inhibitors (44). Additionally, blocking of VEGFR3 signaling using a neutralizing antibody specifically suppresses VEGF-Cinduced lymphangiogenesis in adult mice, but the preexisting lymphatics remained unaffected (45). Similarly, a monoclonal antibody to Nrp2 does not affect established lymphatics in normal adult mice but reduces tumor lymphangiogenesis and inhibits metastasis (18). All these findings support the notion that inactivation of COUP-TFII in the adult may inhibit the growth of newly formed lymphatics exclusively, leaving the preexisting vessels unaffected. Thus, targeting COUP-TFII and/or VEGFR3/ Nrp2 signaling with specific inhibitors in adults may be a safe and potentially beneficial antilymphangiogenic therapeutic strategy. In this regard, the COUP-TFII ligand binding domain structure has been solved recently, and it contains a pocket for binding to small molecule ligands (46). Thus, it is likely that small molecule inhibitors of COUP-TFII that inhibit neo-lymphangiogenesis can be used for cancer treatment.

Primary or inherited lymphedema, the accumulation of proteinrich fluid in the interstitial space, is caused by mutations involved in lymphatic development and function. Although there is currently no cure for lymphedema, the commonly employed treatment is manual lymphatic drainage by massage and external compression (47). Interestingly, a recent study has identified a single mutation in Nrp2 that can cause primary lymphedema in patients (48). Although this mutation occurred in families too small to convincingly confirm cosegregation of mutation with the disease phenotype, it is tempting to speculate that COUP-TFII is another biologically plausible candidate gene associated with clinical lymphedema. Future studies to elucidate the relevance of COUPTFII mutations in lymphedema will be of high clinical interest.

In summary, we identified COUP-TFII as a determinant of lymphatic cell identity and a critical regulator of developmental and postnatal lymphangiogenic response. To our knowledge, COUP-TFII is the first identified transcription factor that directly regulates $\mathrm{Nrp} 2$ expression to modulate the VEGFR3/Nrp2 signaling pathway. The potential role of COUP-TFII in regulating lymphangiogenesis via activation of the VEGFR3/Nrp2 signaling axis enhances the appeal of COUP-TFII as an effective molecular target in pro-lymphangiogenic treatment of primary and secondary lymphedemas or in antilymphangiogenic therapy targeting lymphatic metastasis.

\section{Methods}

Animal experiments. COUP-TFII $/ F$ mice (24), COUP-TFII ${ }^{+/-}$mice, and COUP$T F I I^{-1-}$ embryos (23) were generated as previously reported. The ROSA26CRE$E R^{T 2}$ knock-in mouse strain was provided by T. Ludwig (Columbia University, New York City, New York, USA) (25). All mouse strains, except the mice for the mammary tumor model, were maintained in a mixed genetic background $(129 / \mathrm{Sv} \times \mathrm{C} 57 \mathrm{BL} / 6)$ and received standard rodent chow. A vaginal plug was set as E0.5. For inducible deletions during embryonic stages, Tam was dissolved in corn oil (Sigma-Aldrich; $10 \mathrm{mg} / \mathrm{ml}$ ), and $3 \mathrm{mg}$ Tam was injected intraperitoneally into pregnant females starting at E9.5 to E13.5. To induce COUP-TFII inactivation in the adult, 2 -month-old mice were intraperitoneally injected with $0.5 \mathrm{mg}$ Tam for 5 consecutive days.

For the mammary gland mouse tumor model, MMTV-PyMT mice with a FVB background were obtained from The Jackson Laboratory (49) and were backcrossed to a C57BL/6 background for 7 generations. The backcrossed MMTV-PyMT mice were then crossed with either COUP-TFII ${ }^{F / F}$ or ROSA26CRE-ER ${ }^{T 2}$;COUP-TFII ${ }^{F / F}$ mice on a C57BL/ 6 background to generate MMTV-PyMT;COUP-TFII F/F (PyMT;F/F) or MMTV-PyMT;ROSA26CRE$E R^{T 2}$;COUP-TFII ${ }^{F / F}\left(P y M T\right.$;CRE-ER $\left.{ }^{T 2} /+F / F\right)$ mice. To induce COUP-TFII inactivation in the mouse tumor model, 1-month-old mice were intraperitoneally injected with $0.5 \mathrm{mg}$ Tam for 5 consecutive days.

For all studies, littermate controls were used. Experimental animals and studies were approved by the Institutional Animal Care and Use Committee of Baylor College of Medicine.

Histological analysis and immunohistochemistry. Mouse tissues were fixed with $4 \%$ PFA, dehydrated, and embedded in paraffin. For histological analysis, sections $(7 \mu \mathrm{m})$ were stained with H\&E. For immunohistochemistry and whole-mount immunofluorescence staining, we used standard methods, following protocols detailed in the Supplemental Methods. The following antibodies were used: anti-COUP-TFII (Perseus Proteomics); anti-EphB4, anti-VEGFR3, anti-Neuropilin-1, anti-Neuropilin-2, and anti-Endoglin (R\&D Systems); anti-VEGFR2 (Cell Signaling Technology); anti-Prox1 and anti-LYVE1 (AngioBio); anti-podoplanin (Hybridoma Bank, University of Iowa, Iowa City, Iowa, USA); anti-laminin (DakoCytomation); anti-collagen IV (Abcam); anti-Ki67 (BD Biosciences); and antiCD31 (PECAM) (BD Biosciences).

Lymphangiography. To visualize functional lymphatic vessels in adult mice, $2,000-\mathrm{kDa}$ FITC $(10 \mathrm{mg} / \mathrm{ml}$ in PBS; Sigma-Aldrich) was intradermally injected into the mouse tails or ears, and lymphatic vasculature was immediately monitored by fluorescence microscopy (Zeiss). Alternatively, Evans blue dye $(10 \mathrm{mg} / \mathrm{ml}$ in PBS; MP Biomedicals) was subcutaneously injected into the hind limb foot pads of mice that were first anesthetized with Avertin (2-2-2-tribromoethanol). Mice were perfused with PBS and then photographed.

$R N A$ interference and plasmid construction. Human primary LECs were isolated from neonatal human foreskins as previously described (29). Cells were maintained in an EC-basic medium (EGM; Clonetics) at $37^{\circ} \mathrm{C}$ in a $5 \%$ $\mathrm{CO}_{2}, 95 \%$ humidity incubator. To silence COUP-TFII expression, cells were transfected with COUP-TFII siRNA or control siRNA (Dharmacon), using Oligofectamine (Invitrogen) according to manufacturer's instructions. The siRNA sequences are listed in Supplemental Table 1. For lentivirus construction, human Nrp2 (NM_003872) was constructed by PCR using template plasmids from F. Bono (Sanofi-Aventis Recherche \& Développement, Toulouse, France) and was subcloned in a lentivector with a CMV promoter.

Spheroid-based angiogenesis assay. The angiogenic assay was carried out as described previously (50). Briefly, early passage LECs, subsequent to trans- 
fection or transduction, were suspended and then aggregated overnight to form cellular spheroids (500 cells/spheroid). LEC spheroids were embedded into collagen gels, and then $250 \mathrm{ng} / \mathrm{ml}$ VEGF-C in EGM-2 medium (Lonza) were added on top of the gel. After 24 hours, in vitro angiogenesis was quantified by measuring the number of sprouts and the cumulative length of sprouts that had grown out of each spheroid using NIH ImageJ software (http://rsbweb.nih.gov/ij/). Eleven to thirteen spheroids per experimental group were analyzed.

In vitro proliferation assay. Cell proliferation was measured using a modified MTS assay as previously described (51). In brief, siRNA-treated LECs were incubated at $8 \times 10^{4}$ cells per well in 96-well plates coated with fibronectin $(10 \mathrm{ng} / \mathrm{ml})$ and deprived of FBS for 12 hours, followed by stimulation with or without the indicated concentrations of VEGF-C in cultured medium for 48 hours. Twenty $\mu$ l of MTS tetrazolium compound (CellTiter 96 AQueous Kit; Promega) was added to each well, and the cells were incubated for 4 hours at $37^{\circ} \mathrm{C}$. Cell proliferation is expressed as a percentage of the control.

Wound scratch assay. Human primary LECs after transfection were seeded at high density in a cultured medium, followed 1 day later by a wounding of the confluent monolayer by scraping with a pipette tip.

Quantitative RT-PCR assay. Total RNAs were extracted from LECs using TRIzOL (Invitrogen). First-strand cDNA were synthesized by SuperScriptIII Reverse Transcriptase, using random hexanucleotide primers according to the manufacturer's instructions (Invitrogen). Quantitative RT-PCR analyses were carried out using the ABI PRISM 7500 Fast Real-Time PCR System (Applied Biosystems) and SYBR Green PCR Master Mix (Applied Biosystems). All expression data were normalized to $18 \mathrm{~S}$ rRNA. The primer sequences are listed in Supplemental Table 2.

ChIPs. Human primary LECs were cultured on $315-\mathrm{cm}$ plates and harvested according to the manufacturer's instructions (Upstate Bio- technology). Chromatin was prepared and immunoprecipitated using an anti-COUP-TFII antibody (Perseus Proteomics), anti-Sp1 antibody (Millipore), and an isotype-matched negative control. Standard PCR to confirm ChIP of the promoter element was carried out using the primers listed in Supplemental Table 3.

Statistics. Results are presented as mean \pm SEM. Statistical analysis was carried out by Student's $t$ test. $P$ values of less than 0.05 were considered significant.

\section{Acknowledgments}

We thank T. Ludwig for providing ROSA26CRE-ER ${ }^{T 2}$ mice, F. Bono for the plasmids, and W. Chen, W. Qien, and X.-F. Tong for their technical support. We are grateful to L. Yu-Lee for scientific input and comments on the manuscript. We would also like to acknowledge the Core of the Diabetes and Endocrinology Research Center for growing the lentivirus for us. This work was supported by NIH grants R01 HL076448 (to S.Y. Tsai), R01 HD17379 (to M.-J. Tsai), R01 DK45641 (to M.-J. Tsai), and P01PJ1 DK059820 (to S.Y. Tsai and M.-J. Tsai) and American Heart Association (to $0865371 \mathrm{~F}$ to Y. K. Hong).

Received for publication June 5, 2009, and accepted in revised form January 20, 2010.

Address correspondence to: Sophia Y. Tsai or Ming-Jer Tsai, Department of Molecular and Cellular Biology, Baylor College of Medicine, One Baylor Plaza, Houston, Texas 77030, USA. Phone: 713.798.6251; Fax: 713.798.8227; E-mail: stsai@bcm.tmc.edu (S.Y. Tsai). Phone: 713.798.6253; Fax: 713.798.8227; E-mail: mtsai@ bcm.tmc.edu (M.-J. Tsai).
1. Alitalo K, Tammela T, Petrova TV. Lymphangiogenesis in development and human disease. Nature. 2005;438(7070):946-953.

2. Adams RH, Alitalo K. Molecular regulation of angiogenesis and lymphangiogenesis. Nat Rev Mol Cell Biol. 2007;8(6):464-478.

3. Srinivasan RS, et al. Lineage tracing demonstrates the venous origin of the mammalian lymphatic vasculature. Genes Dev. 2007;21(19):2422-2432.

4. Wigle JT, et al. An essential role for Prox1 in the induction of the lymphatic endothelial cell phenotype. EMBO J. 2002;21(7):1505-1513.

5. Yaniv K, Isogai S, Castranova D, Dye L, Hitomi J, Weinstein BM. Live imaging of lymphatic development in the zebrafish. Nat Med. 2006;12(6):711-716.

6. Sabin FR. On the origin of the lymphatic system from the veins and the development of the lymph hearts and thoracic ducts in the pig. AmJ Anat. 1902; 1:367-389.

7. Francois M, et al. Sox18 induces development of the lymphatic vasculature in mice. Nature. 2008 456(7222):643-647.

8. Johnson NC, et al. Lymphatic endothelial cell identity is reversible and its maintenance requires Prox 1 activity. Genes Dev. 2008;22(23):3282-3291.

9. Wigle JT, Oliver G. Prox1 function is required for the development of the murine lymphatic system. Cell. 1999;98(6):769-778.

10. Ferrara N, Gerber HP, LeCouter J. The biology of VEGF and its receptors. Nat Med. 2003;9(6):669-676

11. Karkkainen MJ, et al. Vascular endothelial growth factor $\mathrm{C}$ is required for sprouting of the first lymphatic vessels from embryonic veins. Nat Immunol. 2004; 5(1):74-80

12. Karkkainen MJ, Jussila L, Ferrell RE, Finegold DN, Alitalo K. Molecular regulation of lymphangiogenesis and targets for tissue oedema. Trends Mol Med. 2001;7(1):18-22.

13. Karkkainen MJ, et al. Missense mutations interfere with VEGFR-3 signalling in primary lymphoedema. Nat Genet. 2000;25(2):153-159.

14. Irrthum A, Karkkainen MJ, Devriendt K, Alitalo $\mathrm{K}$, Vikkula M. Congenital hereditary lymphedema caused by a mutation that inactivates VEGFR3 tyrosine kinase. Am J Hum Genet. 2000;67(2):295-301.

15. Karpanen T, et al. Functional interaction of VEGF-C and VEGF-D with neuropilin receptors. FASEBJ.2006; 20(9):1462-1472.

16. Favier B, et al. Neuropilin-2 interacts with VEGFR-2 and VEGFR-3 and promotes human endothelial cell survival and migration. Blood. 2006; 108(4):1243-1250.

17. Yuan L, et al. Abnormal lymphatic vessel development in neuropilin 2 mutant mice. Development. 2002 129(20):4797-4806.

18. Caunt $\mathrm{M}$, et al. Blocking neuropilin-2 function inhibits tumor cell metastasis. Cancer Cell. 2008; 13(4):331-342.

19. Shimizu K, et al. Suppression of VEGFR-3 signaling inhibits lymph node metastasis in gastric cancer. Cancer Sci. 2004;95(4):328-333.

20 . He Y, et al. Suppression of tumor lymphangiogenesis and lymph node metastasis by blocking vascular endothelial growth factor receptor 3 signaling. J Natl Cancer Inst. 2002;94(11):819-825

21. Li L, et al. The nuclear orphan receptor COUP-TFII plays an essential role in adipogenesis, glucose homeostasis, and energy metabolism. Cell Metab. 2009;9(1):77-87.

22. You LR, Lin FJ, Lee CT, DeMayo FJ, Tsai MJ, Tsai SY. Suppression of Notch signalling by the COUPTFII transcription factor regulates vein identity. Nature. 2005;435(7038):98-104.

23. Pereira FA, Qiu Y, Zhou G, Tsai MJ, Tsai SY. The orphan nuclear receptor COUP-TFII is required for angiogenesis and heart development. Genes Dev. 1999; 13(8):1037-1049.

24. Takamoto N, et al. COUP-TFII is essential for radi- al and anteroposterior patterning of the stomach. Development. 2005;132(9):2179-2189.

25. de Luca C, et al. Complete rescue of obesity, diabetes, and infertility in $\mathrm{db} / \mathrm{db}$ mice by neuron-specific LEPRB transgenes. J Clin Invest. 2005;115(12):3484-3493.

26. Naiche LA, Papaioannou VE. Cre activity causes widespread apoptosis and lethal anemia during embryonic development. Genesis. 2007;45(12):768-775.

27. Holtwick R, et al. Smooth muscle-selective deletion of guanylyl cyclase-A prevents the acute but not chronic effects of ANP on blood pressure. Proc Natl Acad Sci U S A. 2002;99(10):7142-7147.

28. Lin EY, et al. Progression to malignancy in the polyoma middle $\mathrm{T}$ oncoprotein mouse breast cancer model provides a reliable model for human diseases. Am J Pathol. 2003;163(5):2113-2126.

29. Lee S, et al. Prox 1 physically and functionally interacts with COUP-TFII to specify lymphatic endothelial cell fate. Blood. 2009;113(8):1856-1859.

30. Yamazaki T, Yoshimatsu Y, Morishita Y, Miyazono $\mathrm{K}$, Watabe T. COUP-TFII regulates the functions of Prox 1 in lymphatic endothelial cells through direct interaction. Genes Cells. 2009;14(3):425-434.

31. Pipaon C, Tsai SY, Tsai MJ. COUP-TF upregulates NGFI-A gene expression through an $\mathrm{Sp} 1$ binding site. Mol Cell Biol. 1999;19(4):2734-2745.

32. Kim BJ, Takamoto N, Yan J, Tsai SY, Tsai MJ. Chicken Ovalbumin Upstream Promoter-Transcription Factor II (COUP-TFII) regulates growth and patterning of the postnatal mouse cerebellum. Dev Biol. 2009; 326(2):378-391.

33. Augustin HG, Koh GY, Thurston G, Alitalo K. Control of vascular morphogenesis and homeostasis through the angiopoietin-Tie system. Nat Rev Mol Cell Biol. 2009;10(3):165-177.

34. Tammela T, et al. Angiopoietin-1 promotes lymphatic sprouting and hyperplasia. Blood. 2005; 105(12):4642-4648.

35. Morisada T, et al. Angiopoietin-1 promotes LYVE- 
1-positive lymphatic vessel formation. Blood. 2005; 105(12):4649-4656.

36. Gale NW, et al. Angiopoietin-2 is required for postnatal angiogenesis and lymphatic patterning, and only the latter role is rescued by Angiopoietin-1. Dev Cell. 2002;3(3):411-423.

37. He Y, et al. Vascular endothelial cell growth factor receptor 3-mediated activation of lymphatic endothelium is crucial for tumor cell entry and spread via lymphatic vessels. Cancer Res. 2005;65(11):4739-4746.

38. Lin J, et al. Inhibition of lymphogenous metastasis using adeno-associated virus-mediated gene transfer of a soluble VEGFR-3 decoy receptor. Cancer Res. 2005;65(15):6901-6909.

39. Krishnan J, et al. Differential in vivo and in vitro expression of vascular endothelial growth factor (VEGF)-C and VEGF-D in tumors and its relationship to lymphatic metastasis in immunocompetent rats. Cancer Res. 2003;63(3):713-722.

40. Karpanen T, et al. Vascular endothelial growth factor $\mathrm{C}$ promotes tumor lymphangiogenesis and intralymphatic tumor growth. Cancer Res. 2001;
61(5):1786-1790.

41. Burton JB, et al. Suppression of prostate cancer nodal and systemic metastasis by blockade of the lymphangiogenic axis. Cancer Res. 2008; 68(19):7828-7837.

42. Laakkonen $\mathrm{P}$, et al. Vascular endothelial growth factor receptor 3 is involved in tumor angiogenesis and growth. Cancer Res. 2007;67(2):593-599.

43. Roberts N, et al. Inhibition of VEGFR-3 activation with the antagonistic antibody more potently suppresses lymph node and distant metastases than inactivation of VEGFR-2. Cancer Res. 2006; 66(5):2650-2657.

44. Matsui J, Funahashi Y, Uenaka T, Watanabe T, Tsuruoka A, Asada M. Multi-kinase inhibitor E7080 suppresses lymph node and lung metastases of human mammary breast tumor MDA-MB-231 via inhibition of vascular endothelial growth factorreceptor (VEGF-R) 2 and VEGF-R3 kinase. Clin Cancer Res. 2008;14(17):5459-5465.

45. Pytowski B, et al. Complete and specific inhibition of adult lymphatic regeneration by a novel VEGFR-3 neutralizing antibody. J Natl Cancer Inst. 2005; 97(1):14-21.

46. Kruse SW, et al. Identification of COUP-TFII orphan nuclear receptor as a retinoic acid-activated receptor. PLoS Biol. 2008;6(9):e227.

47. Karpanen T, Alitalo K. Molecular biology and pathology of lymphangiogenesis. Annu Rev Pathol. 2008; 3:367-397.

48. Ferrell RE, Kimak MA, Lawrence EC, Finegold DN. Candidate gene analysis in primary lymphedema. Lymphat Res Biol. 2008;6(2):69-76.

49. Guy CT, Cardiff RD, Muller WJ. Induction of mammary tumors by expression of polyomavirus middle $\mathrm{T}$ oncogene: a transgenic mouse model for metastatic disease. Mol Cell Biol. 1992;12(3):954-961.

50. Potente $\mathrm{M}$, et al. Involvement of Foxo transcription factors in angiogenesis and postnatal neovascularization. J Clin Invest. 2005;115(9):2382-2392.

51. Fritz-Six KL, Dunworth WP, Li M, Caron KM. Adrenomedullin signaling is necessary for murine lymphatic vascular development. J Clin Invest. 2008; 118(1):40-50. 